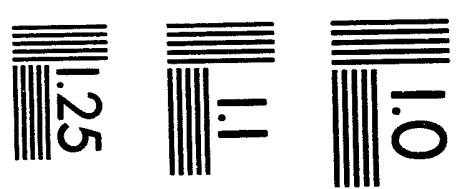

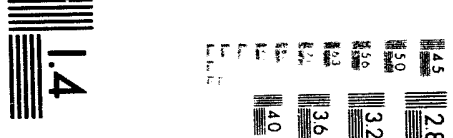

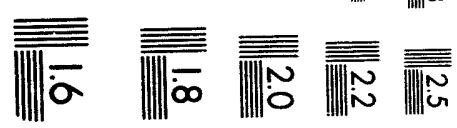



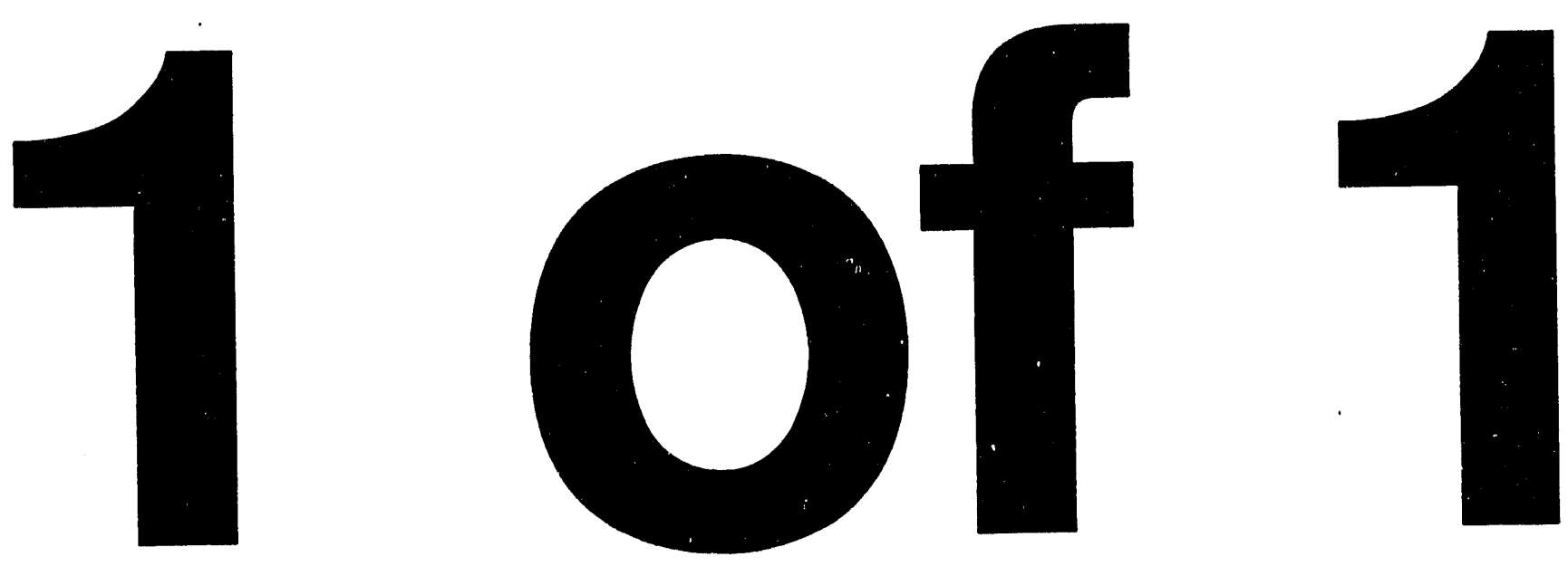
UC-700 and UC-704

Issued: November 1993

Deviatoric Stresses

and Plastic Strain Rates

in Strong Shock Waves for Six Metals

Davis L. Tonks 


\title{
Deviatoric Stresses and Plastic Strain Rates in Strong Shock Waves for Six Metals
}

\author{
by
}

\section{Davis L. Tonks}

\begin{abstract}
The strong shock theory of D. C. Wallace [Phys. Rev. B24, 5597 (1981) and Phys. Rev. B24, 5607 (1981)] is used to calculate the shock structure for $1100 \mathrm{Al}, 2024 \mathrm{Al}, \mathrm{Cu}, \mathrm{Fe}, \mathrm{Ta}$, and $\mathrm{U}$. Emphasis is given to the behavior of plasticity, i.e., average deviatoric stresses, plastic and total strains, and strain rates, which are given in figures for a number of shock strengths. This information will be useful for modeling plasticity in metals under extreme conditions. It was used for part of the PTW model for mechanical behavior.
\end{abstract}




\section{INTRODUCTION}

In recent years Wallace has presented a theory for strong shock waves in metals. ${ }^{1,2}$ This theory incorporates both heat flow and plastic flow as dissipative mechanisms. Heat flow is necessary at the beginning of the shock wave to drive the pressure up to values unattainable by elastic deformation alone. Plastic flow is necessary later on in the shock wave to produce the enormous amounts of heat to do this.

Weak shocks, ${ }^{3,4}$ on the other hand, require only plastic flow to produce the necessary shock steepness, since this is less than that achievable by elastic strains alone. The transition between weak and strong shock waves occurs when the elastic precursor in the weak shock becomes absorbed into the main shock rise as this steepens with shock strength. This condition is called an "overdriven" shock wave.

When the strong shock is steady, as is assumed for most experimentally measured strong shock waves, ${ }^{1}$ the shock velocity can be used to integrate the equations of motion to obtain the normal stress as a function of the volumetric strain. With this behavior known, the plastic flow behavior can be deduced from that of the heat flow which is known under strong shock conditions from fairly reliable extrapolations using solid state physics. ${ }^{1,2}$ Since the shock-velocity / particlevelocity behavior is known experimentally, this means that the plastic flow behavior can be inferred from the known heat flow behavior, the shock-velocity / particle-velocity behavior, and the known thermoelastic behavior of the material. The latter is not known as well as one would like under Mbar shock conditions, but is known well enough to make estimates.

Using this theory, the deviatoric stresses, (deviatoric) plastic strains, and plastic strain rates can, in principle, be found. The thermoelastic behavior under Mbar shock conditions is not known well enough to calculate the deviatoric stress continuously through the shock path, but an estimate of its average through the shock path can be made. ${ }^{1,2}$ This estimate is based on the heating effect of plastic work. The averaged deviatoric stress can then be used to calculate approximate time and space increments through the shock path from which approximations to the plastic and total strain rates can be obtained.

The purpose of this report is to carry out the above calculation for six metals: $1100 \mathrm{Al}, 2024$ $\mathrm{Al}, \mathrm{Cu}, \mathrm{Fe}, \mathrm{Ta}$, and $\mathrm{U}$. The focus here is on the plastic behavior in terms of the average deviatoric stress, plastic and total strains, and strain rates. These will be reported in figures. The heat flow and entropy are not so reported but can be reproduced, if needed, using the equations given herein.

The emphasis here is on the calculational details rather than the general theory which is described adequately in Refs. 1 and 2 . The equations presented here will provide the reader with a reasonable idea of the general theory, however.

Section II contains an enumeration of the equations that were integrated to obtain the information in the figures. Some explanation of their physical basis is given. Section III contains the results for each metal, together with a listing of the parameters used for each in the calculation. 


\section{Equations Used and the Underlying Physical Basis}

The equations given here were supplied to the author by Wallace. ${ }^{5}$ Most appear in the published papers, ${ }^{1,2}$ but those involving the linear approximation for the shock velocity-particle velocity do not.

The integration through the shock path depends on an approximation to the true path in a plot of the temperature, $T$, versus the compression, $\varepsilon$. The approximate path is pictured in Fig. 1, which is reproduced from Fig. 1 of Ref. 2. ${ }^{2}$ This approximation was found by investigating rigorous bounds on this path of temperature versus $\varepsilon$ from the behavior of various ideal materials, e.g., an inviscid fluid. ${ }^{1}$ The approximate path is bounded fairly closely by these considerations and should give realistic results for the present calculations.

The first part of the approximate path of $T$ versus $\varepsilon$ follows that of a nonplastic solid, i.e., one in which only elastic strains and heat conduction occur. This nonplastic-solid path is followed to a certain point, called point $c$, at which plastic flow is assumed to become important. The putil of $T$ versus $\varepsilon$ from point $c$ to the end of the shock wave is taken to be a straight-line from point $c$ to the end point on the Hugoniot, which is known from the experimental shock velocity particle velocity relation. This straight-line portion is closely bounded as described by Wallace in Ref. 2 . $^{2}$ Point $c$ and the straight-line portion are determined by requiring that the line be tangent to the nonplastic solid curve at point $c$.

In Fig. 1 the approximate shock path used for Pt in Ref. 2 is shown. This path follows the line labeled nonplastic solid in Fig. 1 until the point labeled by $\varepsilon_{c}$ is reached. Thereafter, the path follows the straight-line indicated by the dashed line in Fig. 1. Fig. 1 also shows the path of an inviscid fluid, which is an upper bound, and the line whose ordinate is the Hugoniot temperature, which is also an upper bound.

Thus, the integration up the shock path consists of two stages. The first follows the nonplastic solid and the second follows the straight-line portion where plastic flow is occurring. The first part is called the (heat) conduction front and the second is called the flow region.

Before listing the equations of the shock path, the modeling of the thermoelastic description will be described. The product of $\rho \gamma$, where $\rho$ is the density and $\gamma$ is the Gruneisen parameter, is assumed to be constant. Hence, $\rho_{a} \gamma_{a}$, the value of this product for the initial condition, labeled by a, gives $\gamma$ as a function of $\rho$. The ratio of $G / B$, where $G$ is the shear modulus, and $B$ is the bulk modulus, is assumed to be constant. This gives $G$ as a function of $B . B$ on the Rayleigh line can be expressed in the following way when $\rho \gamma=\rho_{a} \gamma_{a}$ is assumed and when the shock velocity, $D$, is given by

$$
D=c+s V
$$

where $\mathrm{V}$ is the particle velocity, and $\mathrm{c}$ and $\mathrm{s}$ are constants:

$$
B=\rho_{a} \gamma_{a} D^{2} \varepsilon(1-\varepsilon)+\rho_{a} c^{2}(1-\varepsilon)\left[1+\left(s-\gamma_{a}\right) \varepsilon\right](1-s \varepsilon)^{-3},
$$

where $\varepsilon$, the compression, is $1-\rho_{a} / \rho$. 


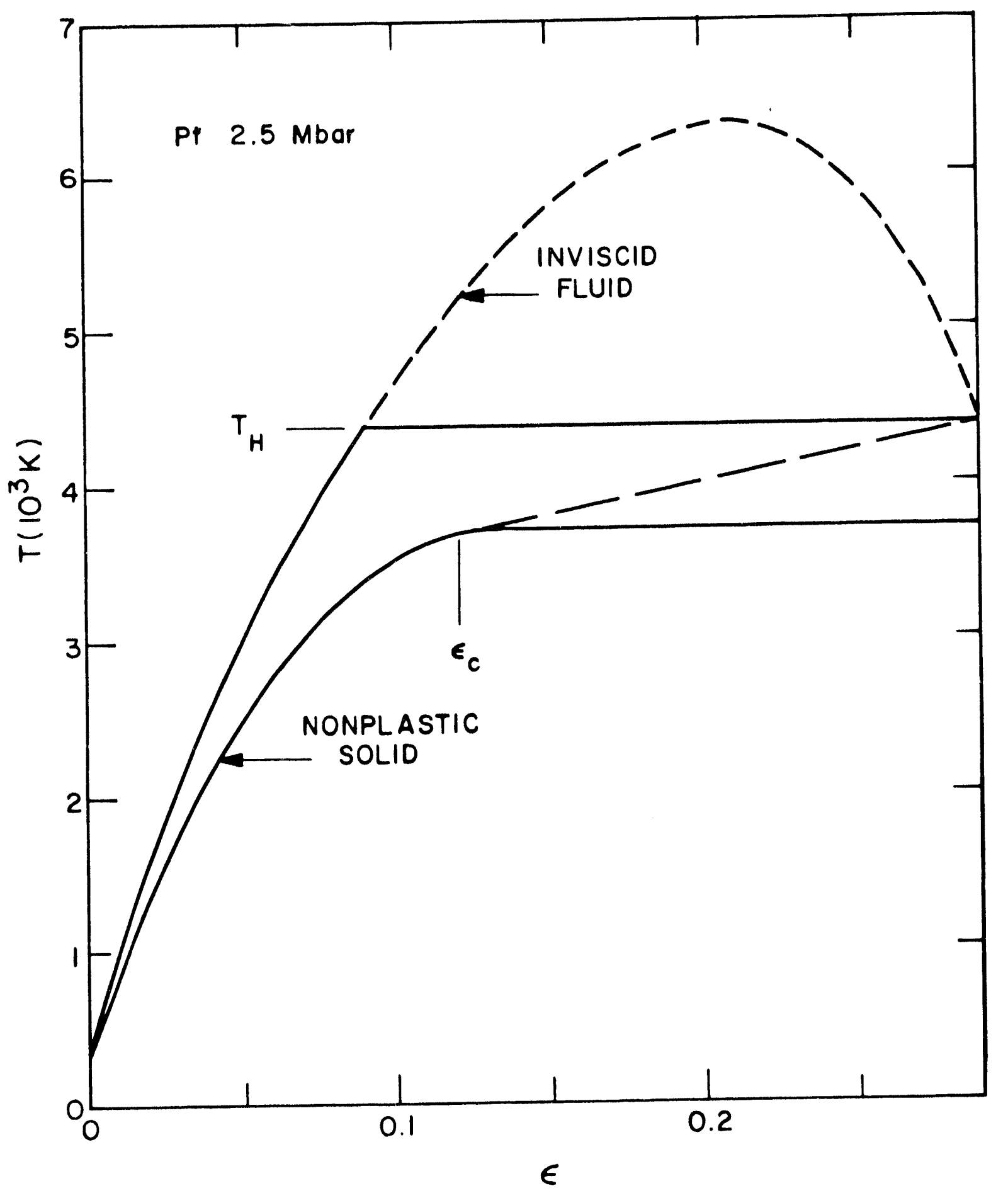

Fig. 1. Upper and lower bounds on the shock path: temperature versus compression. The shock path used here is that of the nonplastic solid followed by the dashed line to the Hugoniot point. 
The assumption that $G / B$ is a constant is made from lack of information. However, it does seem to give reasonable results most of the time. It gives results more reasonable than those of another model (not given here) that included temperature and density dependences, but produced negative (unphysical) strain rates. The calculations are rather sensitive in this manner to $G$.

Wallace's comments on $\mathrm{G}$ as a function of $\mathrm{T}$ and $\mathrm{P}$, pressure, are as follows. ${ }^{5}$ " $G(P, T)$ is diffcult to estimate. The T-dependence of $\mathrm{G}$ is small and easy to estimate, but the $\rho$-dependence is large and cannot be estimated for $\delta \rho / \rho \geq 0.1$. The only way we will get $G(P)$ is by electronic structure calculations, in the distant future. In the meantime, our best estimate for $G$ is still $G / B=$ constant, on the Rayleigh line for overdriven shocks." Support for this is in D. C. Wallace, Phys. Rev. B24, 5607 (1981), Appendix B. ${ }^{2}$

The heat capacity at constant volume, $C_{v}$ is given by

$$
C_{v}=3 N k_{b}+\Gamma T \text {, }
$$

where $3 N k_{b}$ equals $5.9858 \mathrm{cal} / \mathrm{mole} \mathrm{K} . \Gamma T$ is the electronic contribution. $\Gamma$ is given by:

$$
\Gamma(\rho)=\Gamma\left(\rho_{0}\right)\left(\rho_{0} / \rho\right)^{g},
$$

where $\mathrm{g}$ is a constant and $\rho_{0}$ is the density at zero temperature and pressure.

$\Gamma\left(\rho_{0}\right)=\left(\pi^{2} / 3\right) N k_{b}^{2} n\left(\varepsilon_{F}\right)$, where $n\left(\varepsilon_{F}\right)$ is the density of electronic states at the Fermi energy for $\rho=\rho_{0}$. g equals $2 / 3,5 / 3$, and $7 / 3$ for nearly-free-electron metals, $d$-band metals, and f-band metals. ${ }^{6} 3 N k_{b}$ is the lattice contribution to $C_{v}$ and is quite accurate (to $\pm 5 \%$ ). ${ }^{5}$ Further comments by Wallace on the modeling for $C_{1}$, are as follows: "The electronic contribution is a crude approximation, but is the best we can do without extensive electronic structure calculations for each metal. I estimate it to be accurate to $\pm 40 \%$ on the Rayleigh line, for shocks to melting. Note for most cases, the total $C_{\nu}$ will still be quite accurate.

"For $\mathrm{Al}$ and $\mathrm{Cu}, \mathrm{I}$ take $n\left(\varepsilon_{F}\right)$ from "Calculated Electronic Properties of Metals," by V. L. Moruzzi, J. F. Janak, and A. R. Williams (Pergamon, N. Y., 1978). ${ }^{7}$

"Fe undergoes a transition $\alpha \rightarrow \varepsilon$ (bcc to hcp) at $300 \mathrm{~K}$ and 130 kbar. For overdriven shocks, $\mathrm{Fe}$ undergoes two more transitions on the Hugoniot: $\varepsilon \rightarrow \gamma$ (hcp to fcc) at $2.0 \mathrm{Mbar}$, and $\because \rightarrow$ liquid at $2.43 \mathrm{Mbar}$. Hence, $\mathrm{Fe}$ is close-packed in all the overdriven shock regime. Since $n\left(\varepsilon_{F}\right)$ is about the same for $\varepsilon$ and $\gamma$ phases, I ignore the difference. I take $n\left(\varepsilon_{F}\right)$ at $\rho_{0}$ from D. A. Boness, J. M. Brown, and A. K. McMahan, Phys. Earth and Planetary Interiors 42, 227 $(1986)^{8}$

"Ti and U presumably undergo transitions on the Hugoniot to bcc before melting. I am not aware of any information on this. Take $n\left(\varepsilon_{F}\right)$ from the recent work of Olof Eriksson ${ }^{9}$ and also for T'a. 
The figures to come contain values for melt temperatures under various conditions. The model used to calculate them is the following: ${ }^{10}$

$$
T_{m}(\rho)=T_{m}\left(\rho_{s m}\right)\left(\rho / \rho_{s m}\right)^{2 \gamma m-\frac{2}{3}},
$$

where $T_{m}$ is the melt temperature, $\gamma_{m}$ is a special Gruneisen parameter for the melt law, and $\rho_{s m}$ is the density at melt of the solid at zero pressure. The melt temperature does not figure in the calculations along the shock path (Rayleigh line).

The thermal conductivity $\kappa$ can be taken to be a constant for the conditions here. ${ }^{1}$ Wallace's comments on the values chosen are as follows: 5 " $\kappa$ enters only in the time-dependence of the shock process, which it determines completely. I expect T-dependence of $\kappa$ to be negligible, and $\kappa$ to increase with $\rho$ as $\rho$ to $\rho^{2}{ }^{1,2}$ See also my shock theory notes, Section $N$, for more detail. Since $\rho$ nearly doubles at melting on the Hugoniot, the $\rho$-dependence of $\kappa$ is worth including in the estimate. Do this by taking, for $\kappa=$ constant, twice the high-temperature value at normal density; then $\kappa$ should be accurate to within a factor of two for all the shock calculations. Note that, explicitly, all the thermodynamic coefficients are designed to apply in the region between the Hugoniot, and the Rayleigh line for the shock which reaches melting on the Hugoniot."

The shock path calculations depend on knowing the end point on the Hugoniot. This point is determined in the liquid approximation, i.e., only pressure is involved, no deviatoric stresses. For strong shock waves, where the deviatoric stress is small compared to the average stress or pressure, this should be an adequate approximation for the present purposes.

Given $P_{H}$, the pressure on the Hugoniot, one can calculate the quantites $D, \varepsilon_{H}, S_{H},-S_{a}$, , and $T_{H}$, where $S$ is the entropy, by making use of the following equations:

$$
\begin{gathered}
D=c /\left(1-S \varepsilon_{H}\right) \\
P_{H}=\rho_{a} c^{2} \varepsilon_{H} /\left(1-s \varepsilon_{H}\right)^{2} \\
d S_{H}=\frac{c^{2} s \varepsilon_{H}^{2} d \varepsilon_{H}}{T_{H}\left(1-s \varepsilon_{H}\right)^{3}} \\
d T_{H}=\gamma_{a} T_{H} d \varepsilon_{H}+T_{H} d S_{H} / C_{\nu} .
\end{gathered}
$$

The $H$ subscript above refers to the Hugoniot. The last two equations are to be integrated simultaneously up to the point on the Hugoniot. These equations rely on Eq.(1), the linear shockvelocity / particle-velocity relation given earlier, and on the jump conditions through the shock front. $S_{a}$ is the initial entropy.

The following three equations can be integrated simultaneously to produce the path of the nonplastic solid. This portion of the shock path is the conduction front. $J$ is the heat current.

$$
\left.\frac{d J}{\rho_{a} D}=\left\{D^{2}\left[1-\left(1+\frac{4}{3} \frac{G}{B}\right) \gamma_{a} \varepsilon\right]\right\}-c^{2}\left(1+\frac{4}{3} \frac{G}{B}\right)\left[1+\left(s-\gamma_{a}\right) \varepsilon\right](1-s \varepsilon)^{-3}\right\} d \varepsilon / \gamma_{a}
$$




$$
\begin{gathered}
d S=\frac{1}{T} \frac{d J}{\rho_{a} D} \\
d T=\gamma_{a} T d \varepsilon+\frac{1}{C_{\nu}} \frac{d J}{\rho_{a} D} .
\end{gathered}
$$

Eq. (10) arises from the effects of strains and heat on the average stress (pressure). Eq. (11) describes the production of entropy due to the heat flux, the only dissipative mechanism of the nonplastic solid. Eq. (12) describes the change in temperature due to changes in entropy and total strain. All of these equations are specialized to the Rayleigh line.

The point $\mathrm{c}$ on the approximate shock path, where the nonplastic solid behavior ends and the straight-line portion of the plastic flow region begins, is determined by the solution to the following equation:

$$
\left.\frac{d T}{d \varepsilon}\right]_{c}=\frac{T_{H}-T_{c}}{\varepsilon_{H}-\varepsilon_{c}}
$$

where the $\mathrm{c}$ subscript denotes quantities evaluated at point $\mathrm{c}$.

In the flow region, the temperature versus $\varepsilon$ is assumed to have the following behavior:

$$
T(\varepsilon)=T_{1}+X \varepsilon
$$

where $X$ is $\left(T_{H}-T_{c}\right) /\left(\varepsilon_{H}-\varepsilon_{c}\right)$ and $T_{1}$ is $T_{c}-X \varepsilon_{c}$. The following three equations are to be integrated along the straight-line path:

$$
\begin{gathered}
T d S=C_{v}\left(X-\gamma_{a} T\right) d \varepsilon \\
d S=T d S / T \\
d \psi=\left\{\rho_{a} \gamma_{a} T d S+\left[(1-\varepsilon)^{-1}\left(B+\frac{4}{3} G\right)-\rho_{a} D^{2}\right] d \varepsilon\right\} /(2 G),
\end{gathered}
$$

where $B(\varepsilon)$ is given by Eq. (2) and $\psi$ is the plastic strain.

Eq. (17) is based on the effect of entropy and strain on the pressure along the Rayleigh line.

When the above calculation is finished, $\langle\tau\rangle$, the average value of the deviatoric stress (calculated according to the heating effect of plastic work) can be evaluated as follows: ${ }^{2}$

$$
\langle\tau\rangle=\frac{\left(J_{c} / \rho_{a}\right) D+\int_{c}^{H} T d S}{\left(V_{c}+V_{H}\right) \psi_{H}}
$$


where $V_{c}+V_{H}$ equals $\left(2-\varepsilon_{c}-\varepsilon_{H}\right) / \rho_{a}$.

Using $\langle\tau\rangle$, approximate time and space increments can be calculated through the plastic flow region. These can then be used to obtain approximate plastic and total strain rates. This procedure can be accomplished using the following equation:

$$
d Z=(-\kappa) d T /[(1-\varepsilon) J],
$$

which is Eq. (14) from Ref. 2. Here, $d Z$ is the Lagrangian spatial increment. From Eq. (14) (this document), $d T$ can be re-expressed as $X d \varepsilon$. The time increment can be obtained from $d Z$ by dividing it by the shock velocity, D. To evaluate the above expression (19), J is needed. J can be approximately obtained in the flow region by integrating the following modified version of Eq. (5) from Ref. 2 , in which $\langle\tau\rangle$ is substituted for $\tau$ :

$$
d J /\left(\rho_{a} D\right)-T d S-\left(V_{c}+V_{H}\right)<\tau>\mathrm{d} \psi
$$

where $V_{c}$ and $V_{H}$ are the specific volumes at point c and on the the Hugoniot, respectively. The approximate plastic strain rate that cas be obtained from the above equation can be averaged numerically to obtain an average strain rate.

This numerical averaging was done with both plastic strain and compression used for the weight of averaging to produce two versions of the average plastic strain rate through the plastic flow region: $\langle\dot{\psi}\rangle_{\psi}$. and $\langle\dot{\psi}\rangle_{\varepsilon}$ (In the approximate shock path used here, no plastic flow occurs in the conduction front region.)

A rough value of the average plastic strain rate is given by

$$
\langle\dot{\psi}\rangle_{0}=-\left[\frac{\rho_{a} D^{2}}{\kappa \chi} \frac{J_{c} / \rho_{a} D}{A W}\right] \frac{\left(1-\varepsilon_{H}\right)}{\left(\varepsilon_{H}-\varepsilon_{c}\right)} \frac{\psi_{H}}{2},
$$

where AW stands for the atomic weight in $\mathrm{gm} / \mathrm{mole}$. This relation can be obtained from the average of $\psi$ with respect to $\varepsilon$, shown below, by applying certain approximations.

$$
\langle\dot{\psi}\rangle=\int_{c}^{H} \dot{\psi} d \varepsilon /\left(\varepsilon_{H}-\varepsilon_{c}\right) .
$$

Equation (21) can be obtained from the above by the following steps. First, replace $\psi$ in the integrand by $\mathrm{d} \psi / \mathrm{dt}$. Then substitute for $d t$ the form $-(\kappa X d \varepsilon) /(D(1-\varepsilon) J)$ described above. Then, in this expression, replace $\mathrm{J}$ by $\mathrm{J}_{\mathrm{c}} / 2$, its approximate average through the path of integration. Next, replace $1-\varepsilon$ by its approximate average, $\left(1-\varepsilon_{H}\right)$, and finaily, replace $\int_{c}^{H} d \psi / d \varepsilon$ by $\psi_{H}^{\prime}\left(\varepsilon_{H}-\varepsilon_{c}\right)$ 
The units intended for use in all of the above equations are the following. $\mathrm{cm} / \mu \mathrm{s}$ for $\mathrm{D}$; Mbar for stress; cal/mole K for entropy; Kelvin for temperature; cal $/$ mole for $d J /\left(\rho_{a} D\right)$; and $\mathrm{cal} / \mathrm{s} . \mathrm{cm}$ for $\kappa X$. When these units are used, $\dot{\psi}$ comes out in units of $10^{12} / \mathrm{s}$, which will be used in the figures.

\section{Results}

Fig. 2 for Pt is reproduced here from Ref. 2 to show typical results through the shock front for the temperature, entropy, $\tau,\langle\tau\rangle$, and $\psi$ a function of $\varepsilon$ for a shock strength of $0.5 \mathrm{Mbar}$. The vertical line labeled $\varepsilon_{c}$ divides the (heat) conduction front from the plastic flow region. $\tau$ can be calculated in the former but only its average can be obtained in the latter, as mentioned previously. Plastic flow is assumed to occur only in the flow region.

The remaining figures are arranged for the display and interpretation of the average deviatoric stress $\langle\tau\rangle$, obtained by the heat of plastic work. This quantity is, in effect, averaged with respect to plastic strain through the plastic region. The other quantities are given to help understand the conditions under which $<\tau>$ arose. For consistency, they should also be averages tr rough the plastic flow region. However, in preliminary calculations, when the quantities $\mathrm{G}, \mathrm{P}, \mathrm{T}$, and $T_{m}$ were averaged with respect to $\psi$ and $\varepsilon$, the results differed by only a few percent from the values taken at the point along the shock path where one-half of the final plastic strain is achieved. Based on this similarity, for simplicity, all quantities given to help interpret $\langle\tau\rangle$ are taken at this point, labeled by $\mathrm{d}$. They can be considered to be very good approximations to averages through the plastic flow region. Hugoniot pressures and total strains are also given in figures to show what shock strengths are involved.

As described earlier, the plastic strain rate was averaged three different ways. The first two, yielding $\langle\dot{\psi}\rangle_{\varepsilon}$ and $\langle\dot{\psi}\rangle_{\psi}$, involve averaging approximate strain rate values through the shock front using total and plastic strains as weighting factors. These two versions are seen in the remaining figures to be similar. The third way produced $\langle\dot{\psi}\rangle_{0}$ of Eq. (21), which is an approximate version of $\langle\dot{\psi}\rangle_{\varepsilon}$. In the figures, this version is seen to depart from the other two for weaker shock waves. All three of these versions depend upon using $\langle\tau\rangle$ in place of $\tau$ itself in Eq. (20) describing dissipation, since $\tau$ itself cannot be calculated with sufficient accuracy. ${ }^{2}$

In the figures, the label $\log \langle d \psi / \mathrm{dt}>$ on the $\mathrm{y}$-axis is used to denote these three averages.

Table 1 gives the values of physical parameters used to generate the remaining figures.

The $\gamma_{a}$-value used for Fe (2.5) in the strong shock condition is the upper bound suggested by Brown and McQueen. ${ }^{13}$ This $\gamma$-value is close to that of the liquid state, whose $\gamma$-value is expected to be approximately that of the close-packed phases of Fe shocked above the transition to the $\varepsilon$-phase at $130 \mathrm{kbar}$. When the value 1.7, the lower bound of Brown and McQueen, was used in the calculation, negative strain rates resulted due to the temperature at point $c$ (at the end of the conduction-front portion) being already higher than that on the Hugoniot. The slope of the temperature versus $\varepsilon$ plot was negative for the plastic flow regime. This indicates that 1.7 is too low for stiong shock waves.

The values used for $G / B$ are those under ambient conditions. At first sight, values pertaining to high compressions and temperatures might seem more appropriate. However, it is very important in the calculation to accurately calculate the (initial) thermal conduction portion of the shock 


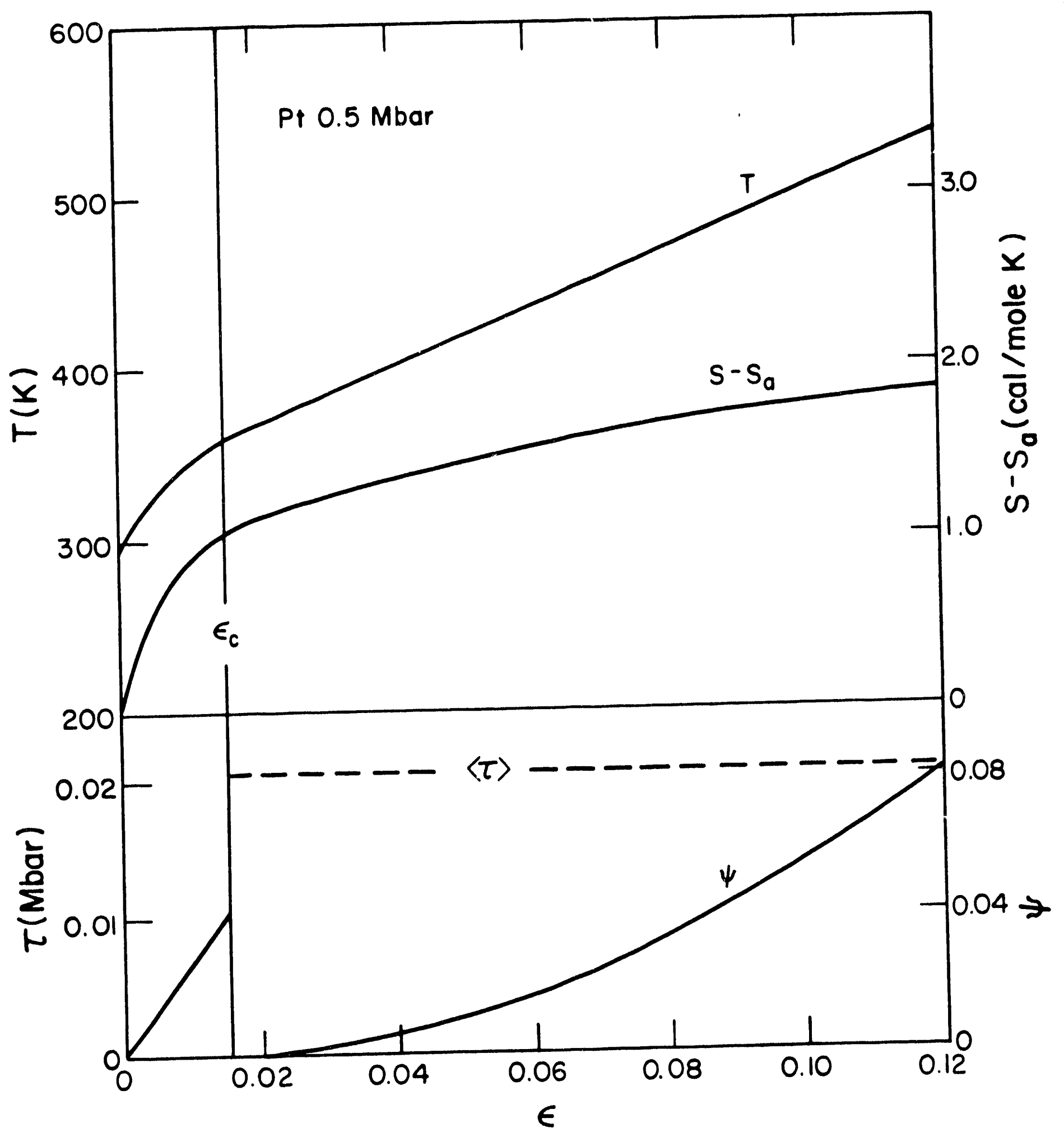

Fig. 2. Temperature, T; entropy, S; plastic strain, $\psi$; and deviatoric stress, $\tau$ plotted versus compression, $\varepsilon$; calculated for a 0.5 Mbar shock in Pt. The dotted line gives the average $\tau$ in the flow region. 
path to get an accurate temperature at point $c$, where the plastic flow portion begins. This temperature value, together with the temperature on the Hugoniot, determine the plastic flow. It is the $G$ / $B$ behavior closer to ambient conditions that is important for the conduction front and for the temperature at point $\mathrm{c}$. When values for $G / B$ appropriate for strong compressions, i.e., lower values, were tried in the calculation, negative plastic strain rates resulted due to $T_{c}$ being already higher than the temperature on the Hugoniot. .

\begin{tabular}{|l|l|l|l|l|l|l|}
\hline Quantity & $1100 \mathrm{Al}$ & $2024 \mathrm{Al}$ & $\mathrm{Cu}$ & $\mathrm{Fe}$ & $\mathrm{Ta}$ & $\mathrm{U}$ \\
\hline \hline $\mathrm{AW}(\mathrm{gm} / \mathrm{mole})$ & 27.0 & 27.0 & 63.54 & 55.85 & 180.948 & 238.04 \\
\hline$c(\mathrm{~cm} / \mu \mathrm{s})$ & $0.5386^{11}$ & $0.5328^{12}$ & $0.3933^{11}$ & $0.3955^{13}$ & $0.3293^{11}$ & $0.2487^{14}$ \\
\hline $\mathrm{s}$ & $1.339^{11}$ & $1.338^{12}$ & $1.50^{11}$ & $1.580^{13}$ & $1.307^{11}$ & $2.20^{14}$ \\
\hline$\rho_{\alpha}\left(\mathrm{gm} / \mathrm{cm}^{3}\right)$ & $2.713^{11}$ & 2.785 & $8.933^{10}$ & $7.87^{10}$ & $16.75^{10}$ & 19.07 \\
\hline$\gamma_{\alpha}$ & 2.0 & 2.0 & 2.0 & $2.5^{13}$ & 1.7 & 2.1 \\
\hline $\mathrm{G} / \mathrm{B}$ & 0.34 & 0.34 & 0.35 & 0.5 & 0.36 & 0.75 \\
\hline$\rho_{0}\left(\mathrm{gm} / \mathrm{cm}^{3}\right)$ & $2.734^{10}$ & $2.734^{10}$ & $9.02^{10}$ & $7.92^{10}$ & $16.8^{10}$ & 19.2 \\
\hline$\Gamma\left(\rho_{0}\right)$ & 2.3 & 2.3 & 1.6 & 11.3 & 6.8 & 12.4 \\
\hline$\left(10^{-4} \mathrm{cal} / \mathrm{moleK}^{2}\right)$ & & & & & & \\
\hline $\mathrm{g}$ & $2 / 3^{6}$ & $2 / 3^{6}$ & $2 / 3^{6}$ & $5 / 3^{6}$ & $5 / 3^{6}$ & $7 / 3^{6}$ \\
\hline$\rho_{\mathrm{sm}}\left(\mathrm{gm} / \mathrm{cm}^{3}\right)$ & $2.542^{10}$ & $2.542^{10}$ & $8.440^{10}$ & $7.254^{10}$ & 15.3 & 17.5 \\
\hline$T_{m}\left(\rho_{\mathrm{sm}}\right)(\mathrm{K})$ & $933.0^{10}$ & $933.0^{10}$ & $1357.0^{10}$ & $1809.0^{10}$ & $3287.0^{10}$ & 1407.0 \\
\hline$\gamma_{m}$ & $2.2^{10}$ & $2.2^{10}$ & $2.0^{10}$ & $1.7^{10}$ & $1.6^{10}$ & 2.1 \\
\hline$\kappa(\mathrm{cal} / \mathrm{s} \mathrm{cm} \mathrm{K})$ & 1.1 & 1.0 & 1.8 & 0.2 & 0.3 & 0.2 \\
\hline
\end{tabular}

Table 1: Material Parameters Used in Calculation Through Shock Front. $\rho_{o}$ is at zero temperature and pressure. The initial temperature for the shock front calculation was taken to be $293 \mathrm{~K}$. All unreferenced values are private communications from $D$. C. Wallace.

To assess the sensitivity of the results to the values of $G / B$ and $\gamma$, these values were varied about the standard values in Table 1 . Changing $G / B$ by $10 \%$ produced the variation in $\langle\tau\rangle / G_{d}$ of 5 to $10 \%$ and produced the variation in $\log \langle\dot{\psi}\rangle_{\varepsilon}$, of a few percent. An exception occured for $\mathrm{Cu}$ for the strongest shock calculated, for which the $10 \%$ change in $\mathrm{G} / \mathrm{B}$ produced a $4 \%$ variation in $\langle\dot{\psi}\rangle_{\varepsilon}$. Changing $\gamma$ by $10 \%$ produced the variation in $\langle\tau\rangle / G_{d}$ of 2 through $4 \%$, except for $\mathrm{Fe}$ and Ta for which the $10 \%$ change in $\gamma$ produced the variation in $\langle\tau\rangle / G_{d}$ of 5 through $10 \%$. The $10 \%$ change in $\gamma$ produced for most materials the variation in $\log \langle\dot{\psi}\rangle_{\varepsilon}$ of only a few percent, except for $\mathrm{Cu}$ for the strongest shock calculated, for which this quantity varied by $7 \%$. 
Table 2 gives values of $\varepsilon_{0}$ and $P_{0}$, the compression and pressure at the overdriven threshold, where the strong shock theory breaks down. From this table, the reader can judge how closely the overdriven threshold has been approached in the figures. Except for $\mathrm{Cu}$ and $\mathrm{Ta}$, it was not approached very closely.

\begin{tabular}{|l|l|l|l|l|l|l|}
\hline \multicolumn{1}{|c|}{ Quantity } & $1100 \mathrm{Al}$ & $2024 \mathrm{Al}$ & $\mathrm{Cu}$ & $\mathrm{Fe}$ & $\mathrm{Ta}$ & $\mathrm{U}$ \\
\hline \hline$\varepsilon_{0}$ & 0.127 & 0.127 & 0.116 & 0.143 & 0.136 & 0.133 \\
\hline$P_{0}$ (Mbar) & 0.146 & 0.146 & 0.235 & 0.293 & 0.366 & 0.314 \\
\hline
\end{tabular}

Table 2: $\varepsilon_{0}$ and $P_{0}$, the compression and pressure at the overdriven threshold for the six metals.

Figures 3 through 7 show calculated results for 1100Al. All but Figure 7 involve $\langle\tau\rangle$ and $<\mathrm{d} \psi / d t\rangle$, which are averages through the plastic flow region, and involve quantities labeled with a d-subscript, which are good approximations to averages through the plastic flow region, as discussed earlier. $\langle\tau\rangle / G_{d}$ is presented since $\tau$ is commonly recognized to scale with $G$. Figure 7 gives part of the Hugoniot to show the final strength of the shock waves from which the other quantities were taken. The shock strengths of Fig. 7 are the same ones used in Figs. 3 through 6. The same is true for the corresponding figures for the other materials.

The temperature plots show that the calculations were extended up to the point where melting occurred at the point of half-final plastic strain. Melting occurred sooner than this on the Hugoniot, of course.

Figures 8 through 12 show corresponding results for $2024 \mathrm{Al}$. They strongly resemble those for $1100 \mathrm{Al}$, since the physical properties of the two are similar.

Figures 13 through 18 show results for copper. Note in Fig. 13 that the estimate for $\log \psi$ taken from Eq. (21) departs, for the weaker shock waves near the overdriven threshold, significantly from the two numerical averages. This shows that the estimate of Eq. (21) departs from the other estimates near the overdriven threshold, where the general theory breaks down.

Figure 18 has $\log$ scales on both axes to show that $\langle\mathrm{d} \psi / d t\rangle$ and $\langle\tau\rangle / G_{d}$ roughly obey a power law. The five square symbols lower down are data of Clifton et al. ${ }^{15}$ from pressure-shear plate-impact experiments. Note that these data and the calculated strong shock points seem to approximately follow a power law. This feature offers a way of modeling both sets of data points.

Figures 19 through 23 show results for iron. Note that the estimate (21) for log $\dot{\psi}$ departs radically from the two numerical averages for the lowest shock pressure, which is somewhat close, but not right on top of, the overdriven threshold. The value for $\gamma_{a}$ for iron under ambient conditions produced unphysical results in the calculation. The value appropriate to close-packed phases was used, which is close to that of the liquid. Since iron undergoes phase transitions to close-packed phases at the shock strengths calculated here, the close-packed value is the appropriate one. The error analysis did not show large sensitivity when variations about this value were made. 
Shock Results for 1100 Aluminum

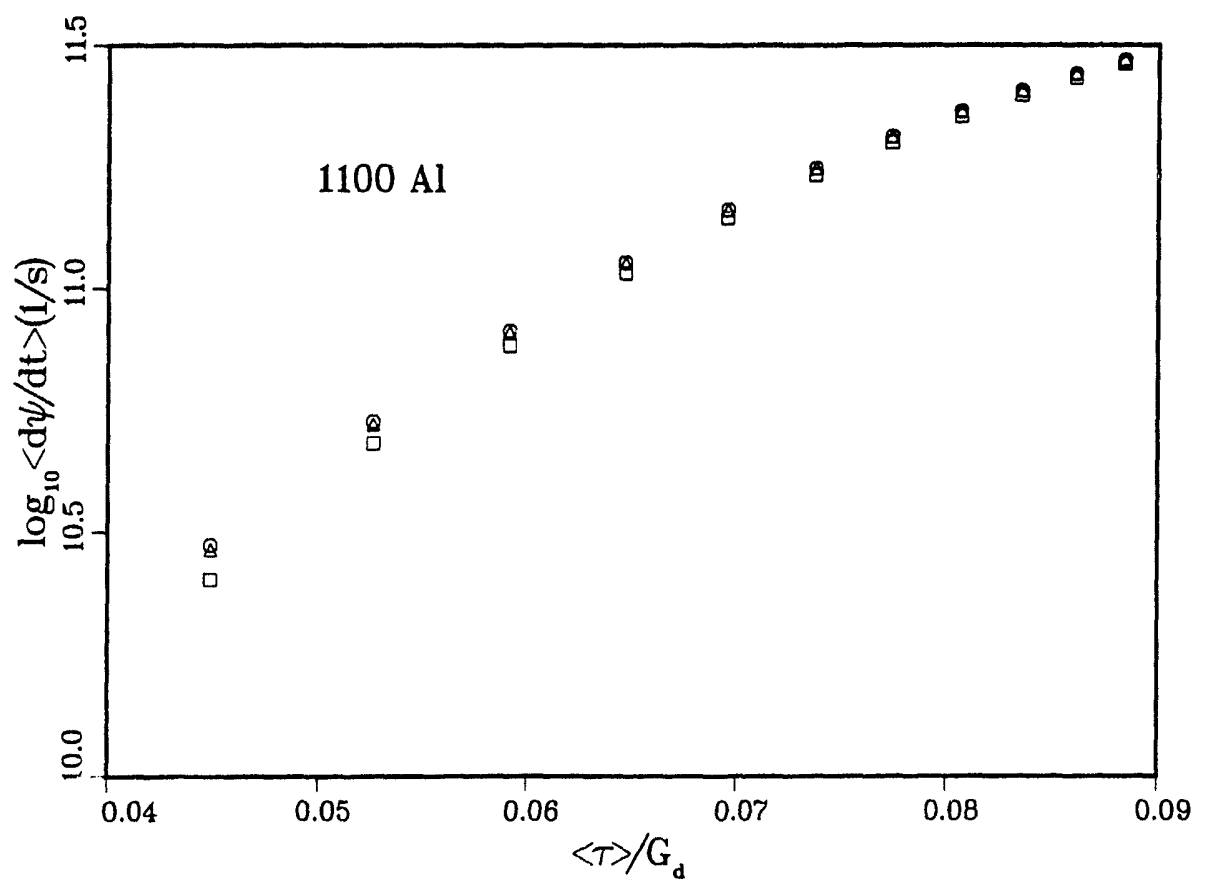

Fig. 3. Average (through shock path) plastic strain rate versus average deviatoric stress divided by $\mathrm{G}_{\mathrm{d}}$ for various shock strengths for $1100 \mathrm{Al}$. The squares, triangles, and circles correspond to strain rates averaged using Eq. (21), averaged numerically using the volumetric strain, and using the plastic strain, respectively.

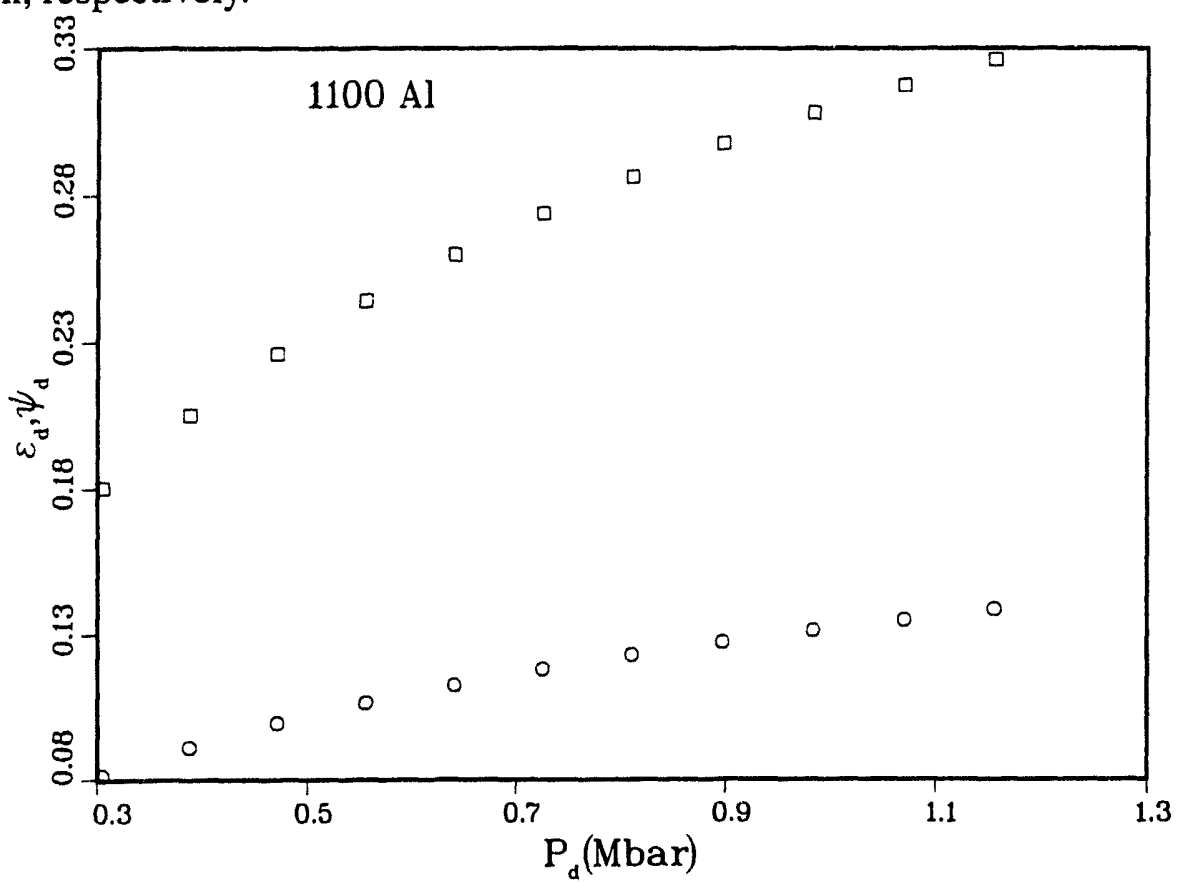

Fig. 4. Plastic strains (circles) and volumetric strains (squares) at the point of half-total plastic strain along the shock path plotted versus the pressure at this point for $1100 \mathrm{Al}$. 


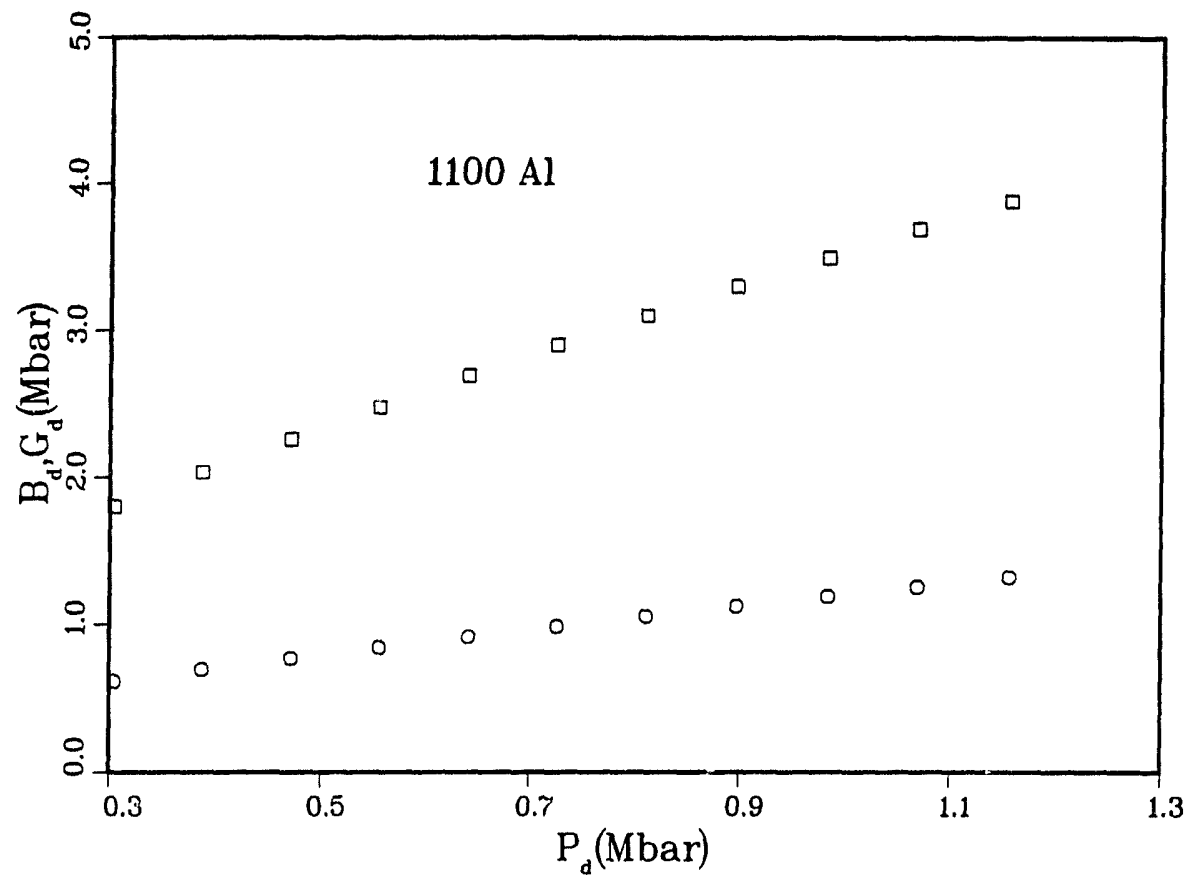

Fig. 5. The shear modulus (circles) and the bulk modulus (squares) at the point of half-total plastic strain plotted versus the pressure at this point for $1100 \mathrm{Al}$.

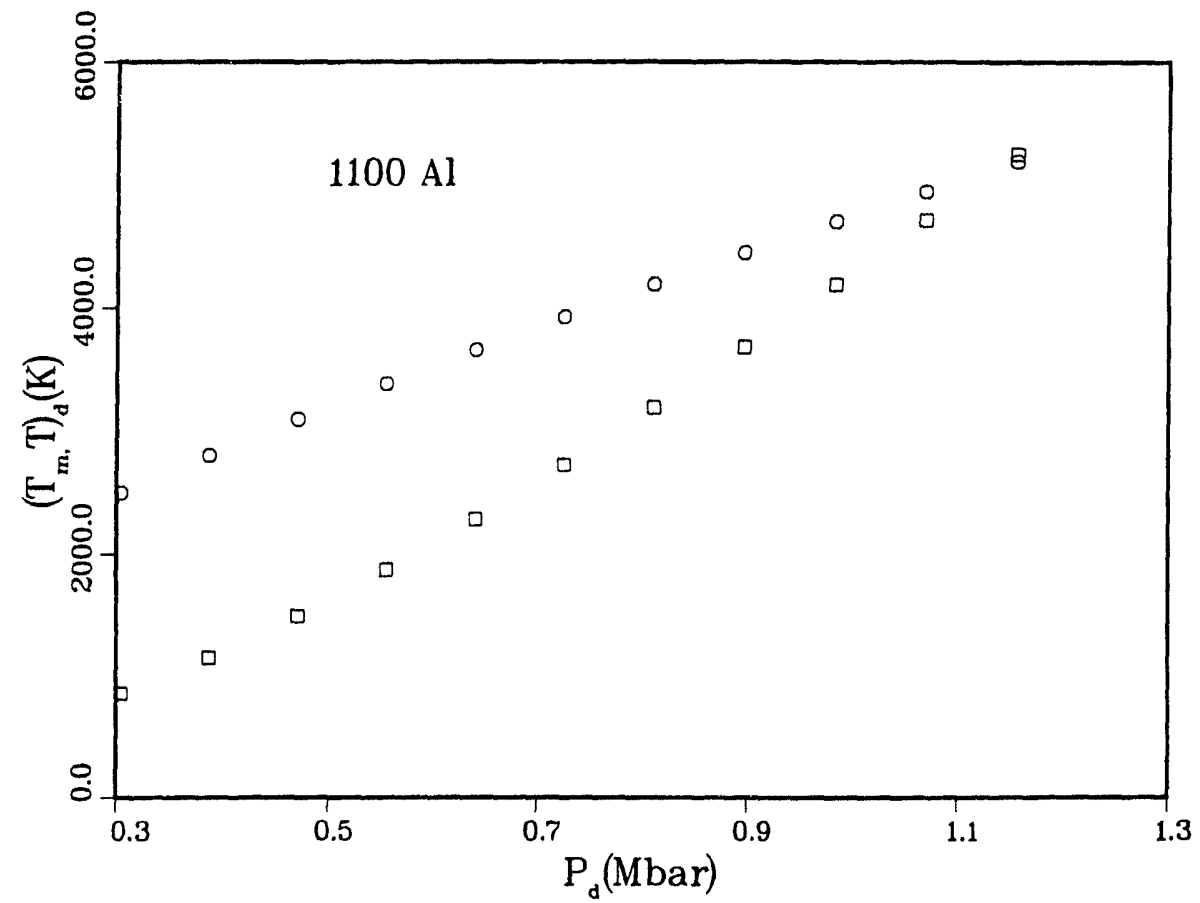

Fig. 6. The melt temperature (circles) and temperature (squares) at the point of half-final plastic strain plotted versus the pressure at this point for $1100 \mathrm{Al}$. 


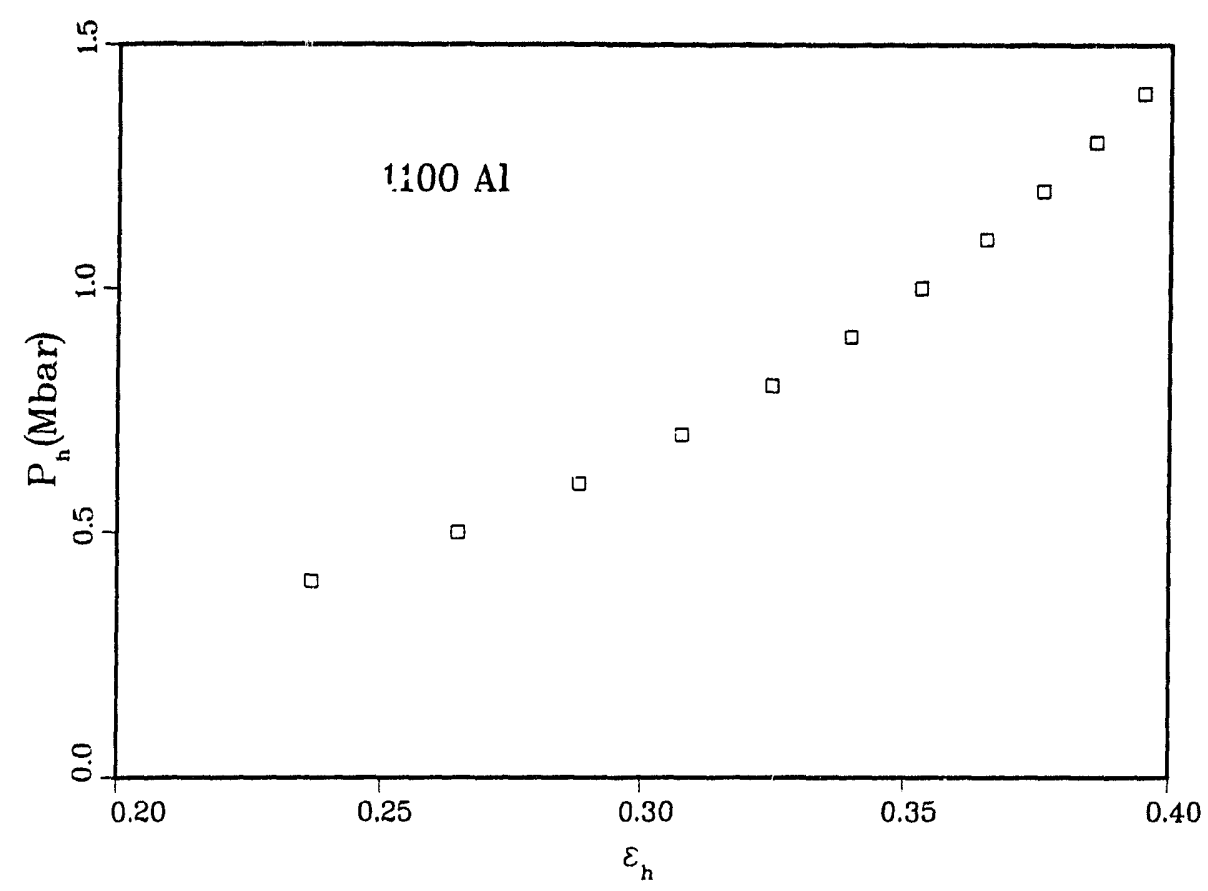

Fig. 7. Hugoniot pressure plotted versus volumetric strain for the same shock waves used to generate the earlier figures for $1100 \mathrm{Al}$. 
Shock Results for 2024 Aluminum

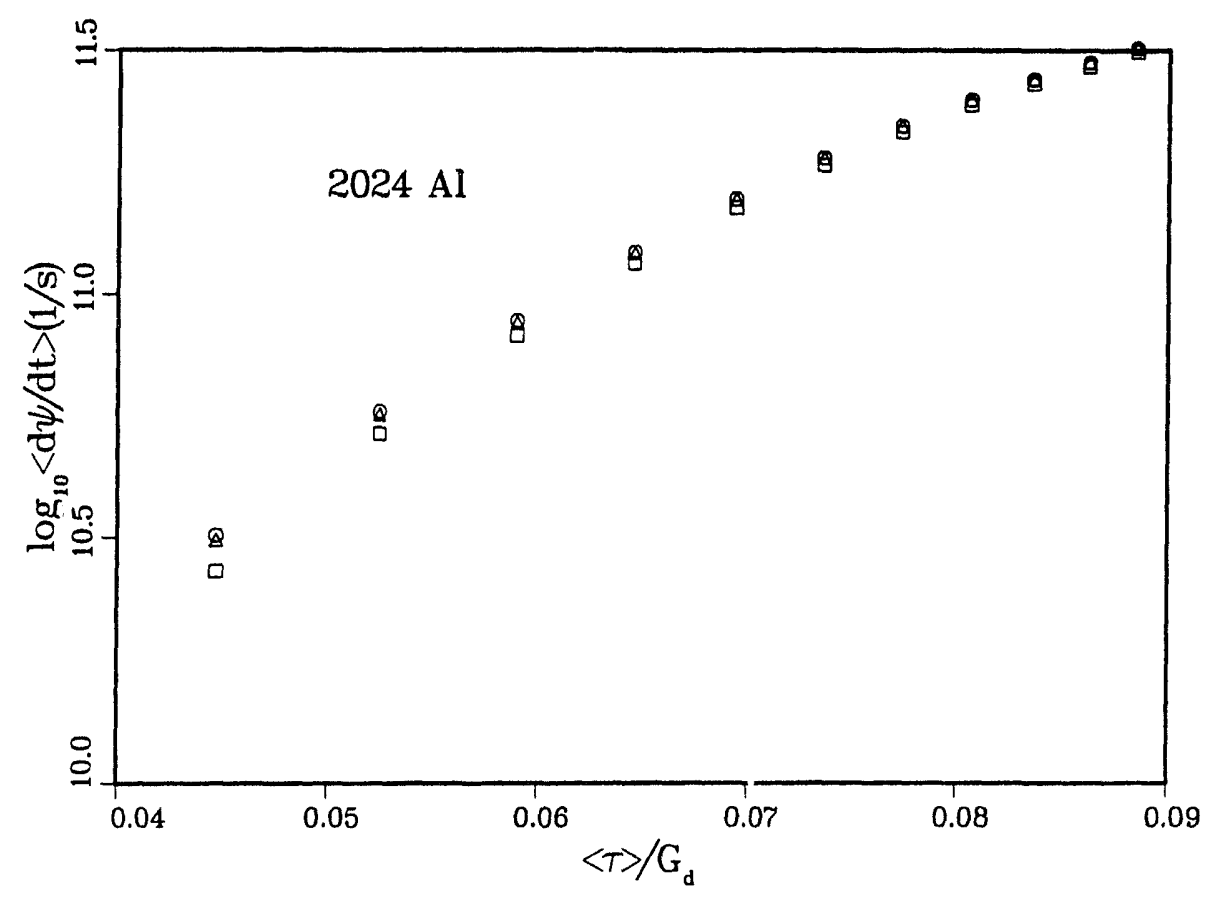

Fig. 8. Average plastic strain rate versus average deviatoric stress divided by $G_{d}$ for various shock strengths for $2024 \mathrm{Al}$. Averages are through the shock path. The squares, triangles, and circles correspond to strain rates averaged using Eq. (21), using the volumetric strain, and using the plastic strain, respectively.

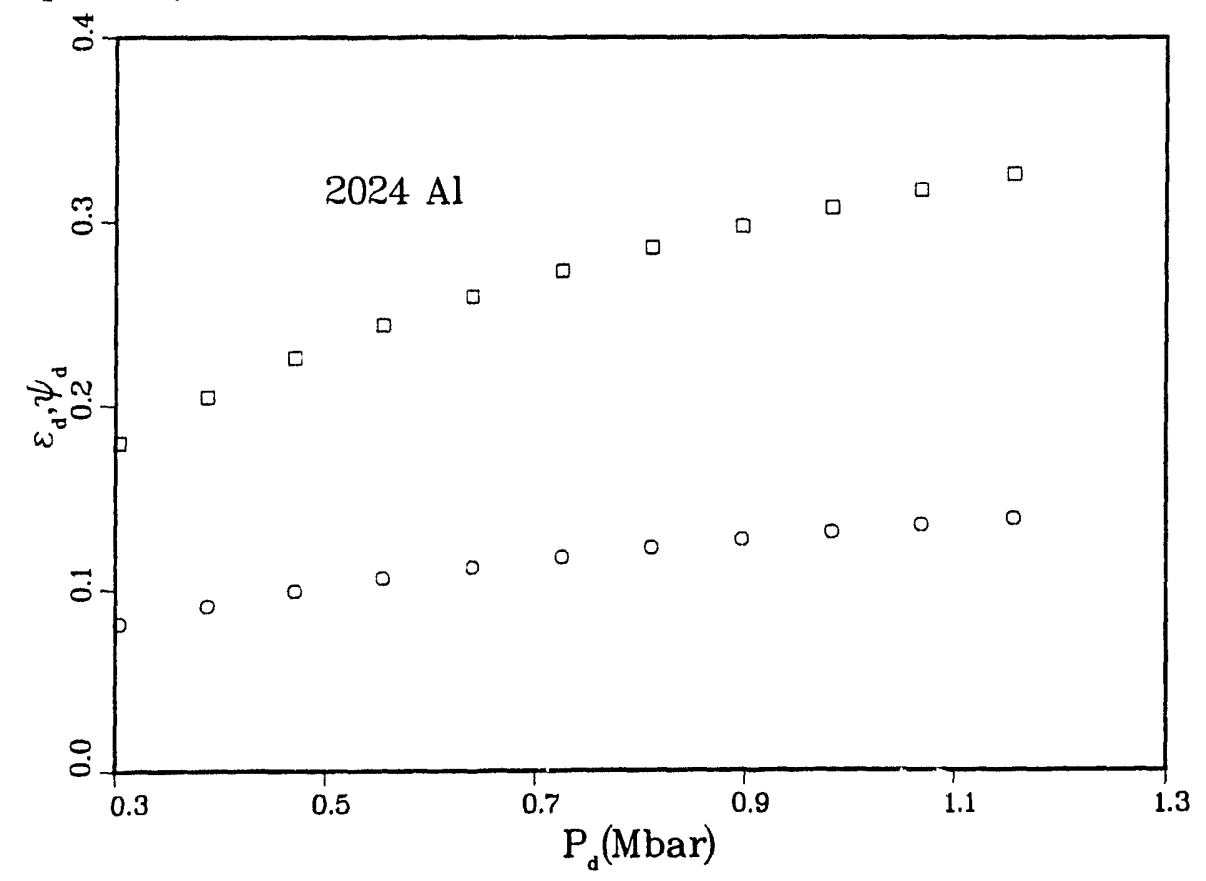

Fig. 9. Plastic strains (circles) and volumetric strains (squares) at the point of half-total plastic strain along the shock path plotted versus the pressure at this point for $2024 \mathrm{Al}$. 


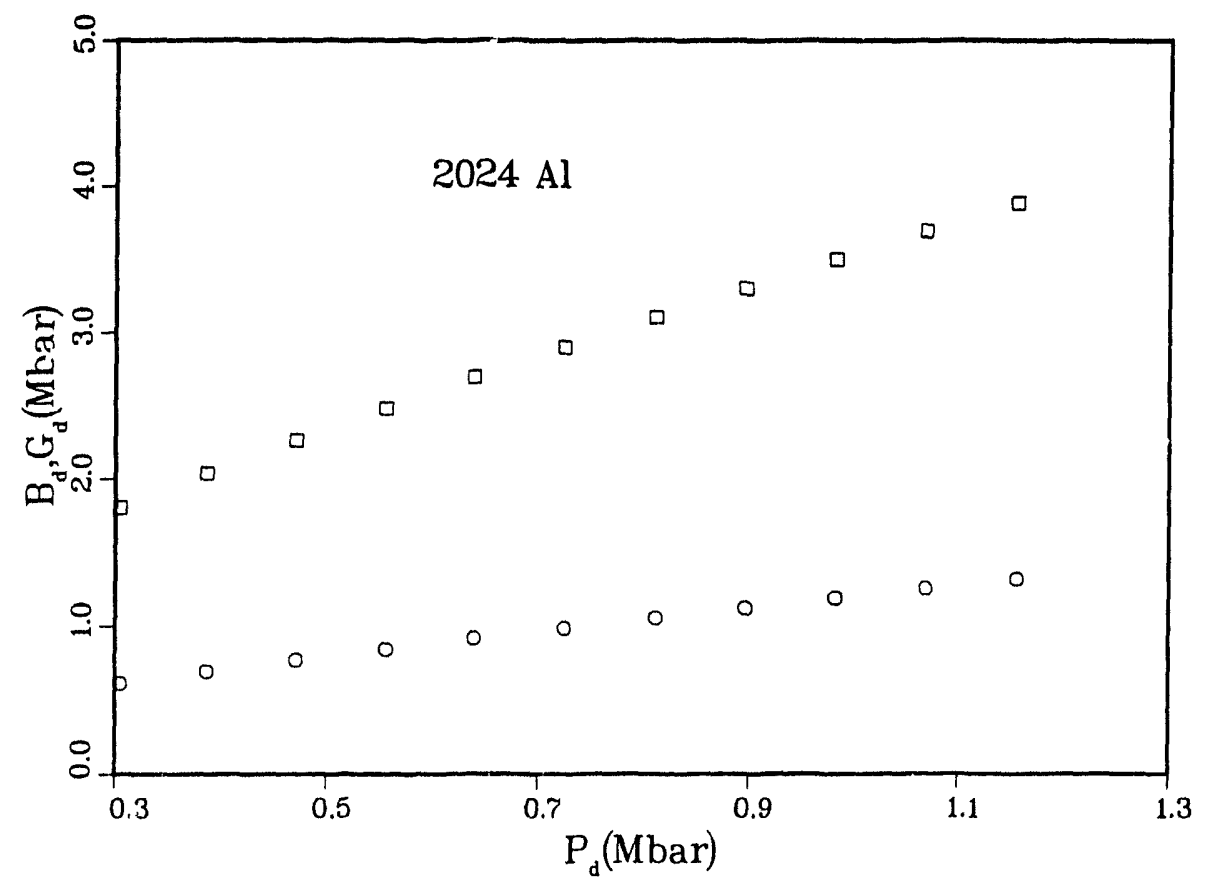

Fig. 10. The shear modulus (circles) and the bulk modulus (squares) at the point of half-final plastic strain plotted versus the pressure at this point for $2024 \mathrm{Al}$.

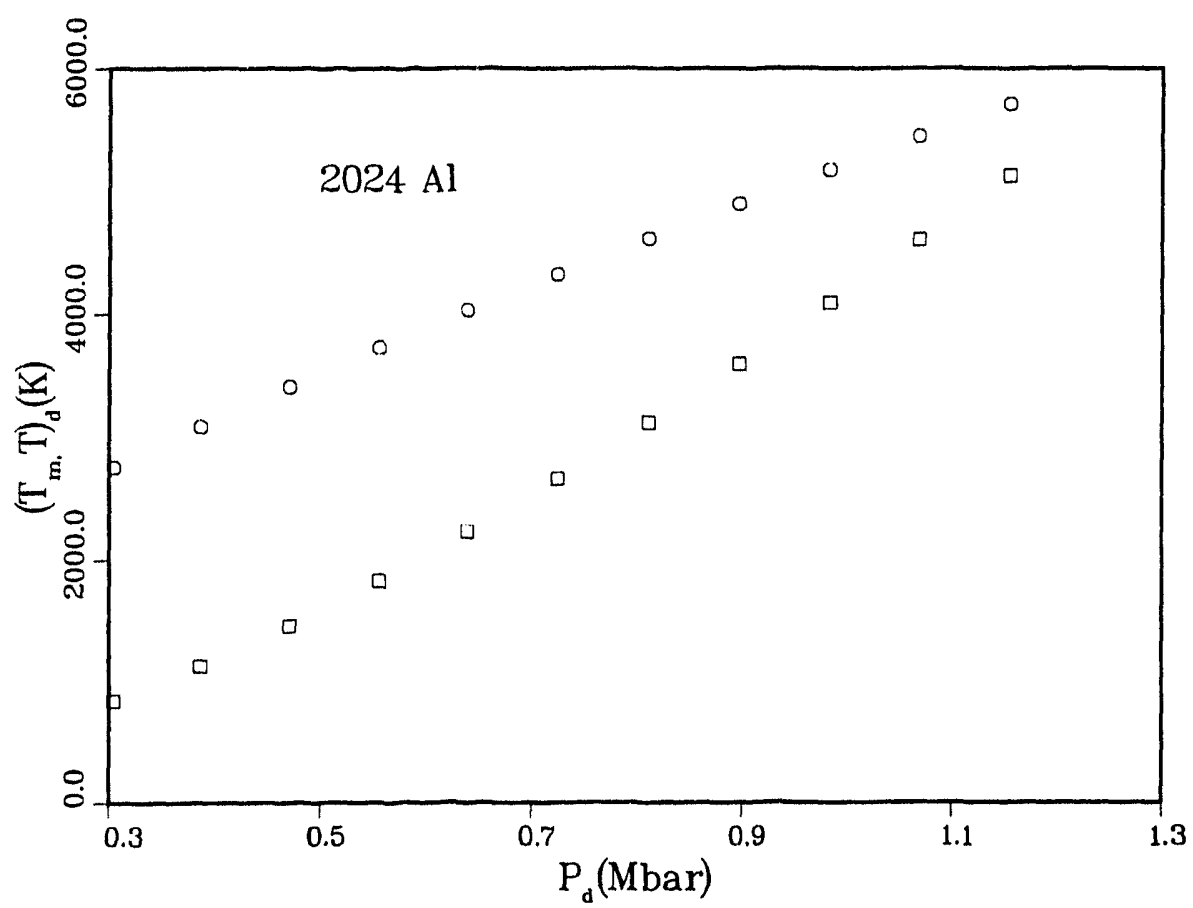

Fig. 11. The melt temperature (circles) and temperature (squares) at the point of half-final plastic strain plotted versus the pressure at this point for $2024 \mathrm{Al}$. 


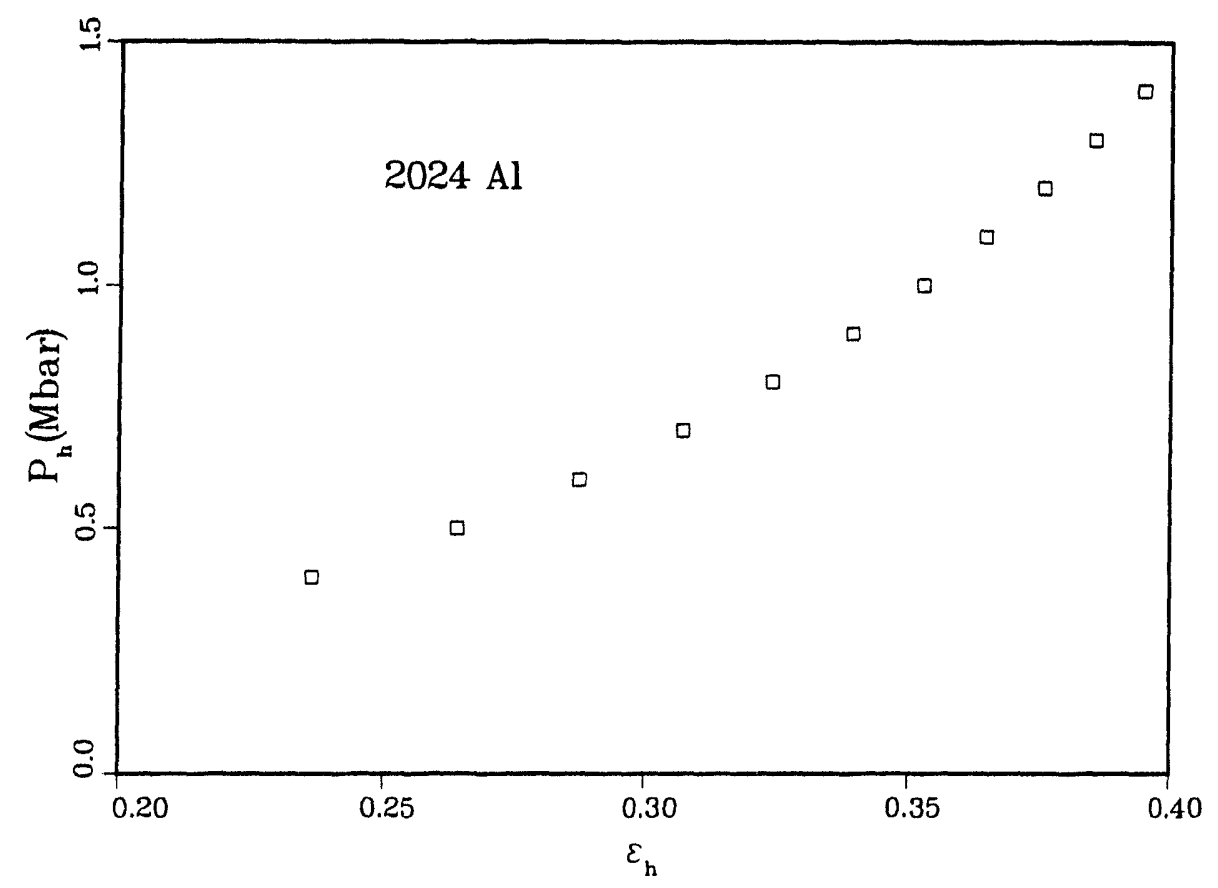

Fig. 12. Hugoniot pressure plotted versus volumetric strain for the same shock waves used to generate the earlier figures for $2024 \mathrm{Al}$. 


\section{Shock Results for Copper}

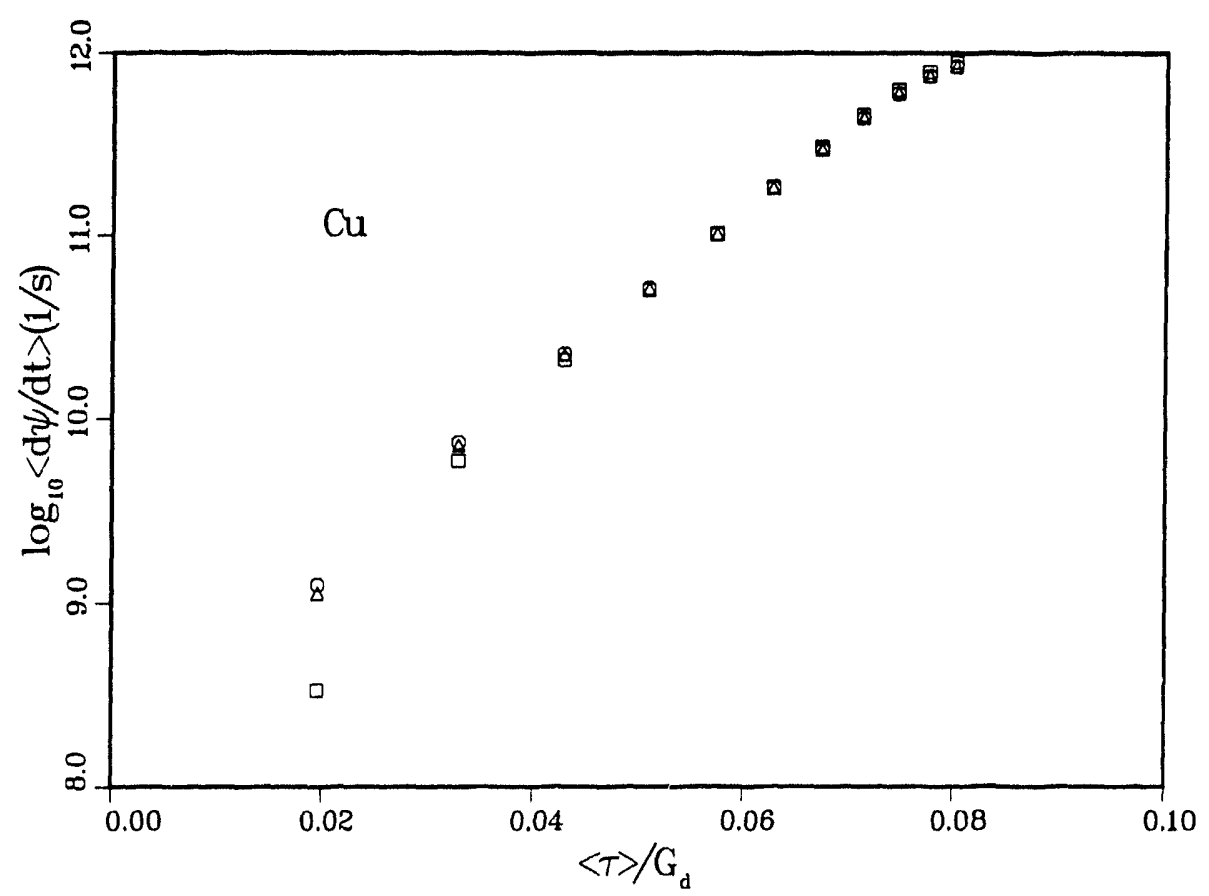

Fig. 13. Average plastic strain rate versus average deviatoric stress divided by $\mathrm{G}_{\mathrm{d}}$ for various shock strengths for $\mathrm{Cu}$. Averages are through the shock path. The squares, triangles, and circles correspond to strain rates averaged using Eq. (21), averaged numerically using the volumetric strain, and using the plastic strain, respectively.

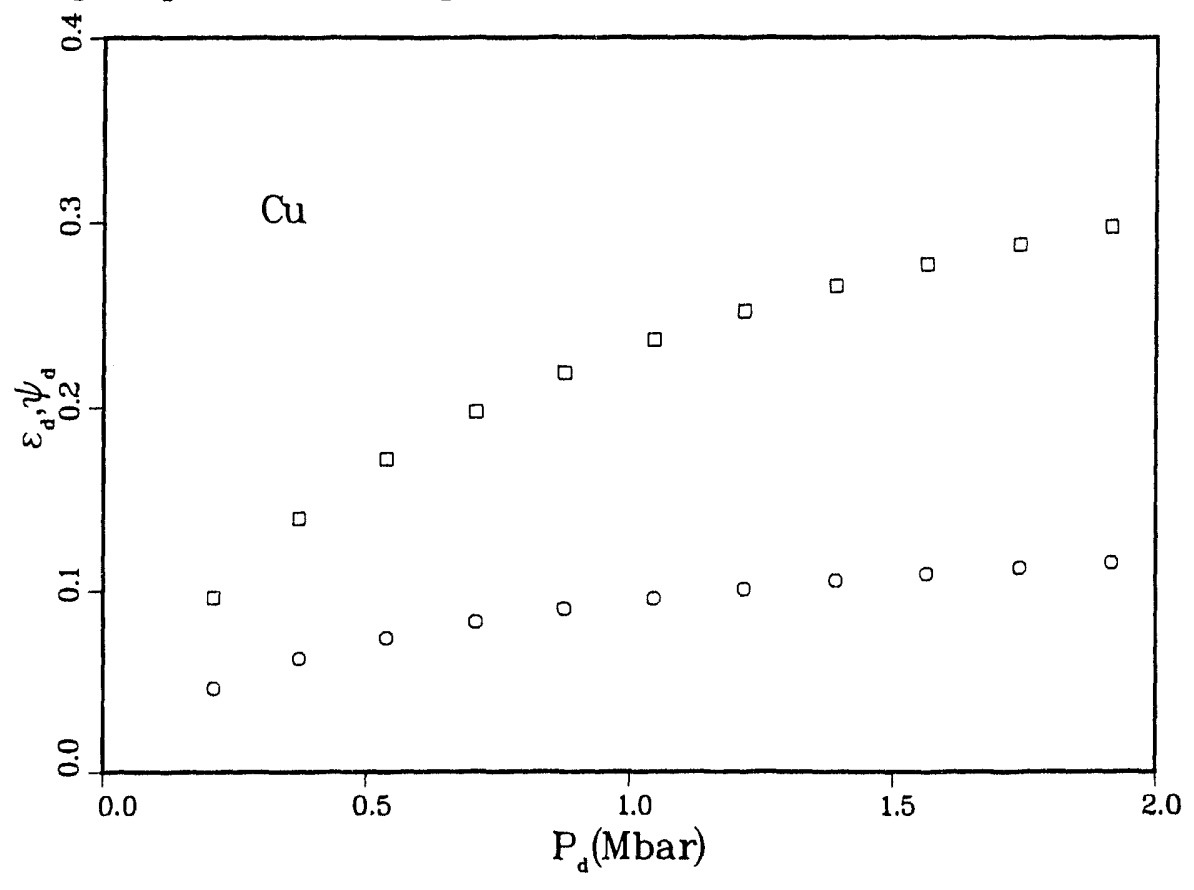

Fig. 14. Plastic strains (circles) and volumetric strains (squares) at the point of half-total plastic strain along the shock path plotted versus the pressure at this point for $\mathrm{Cu}$. 


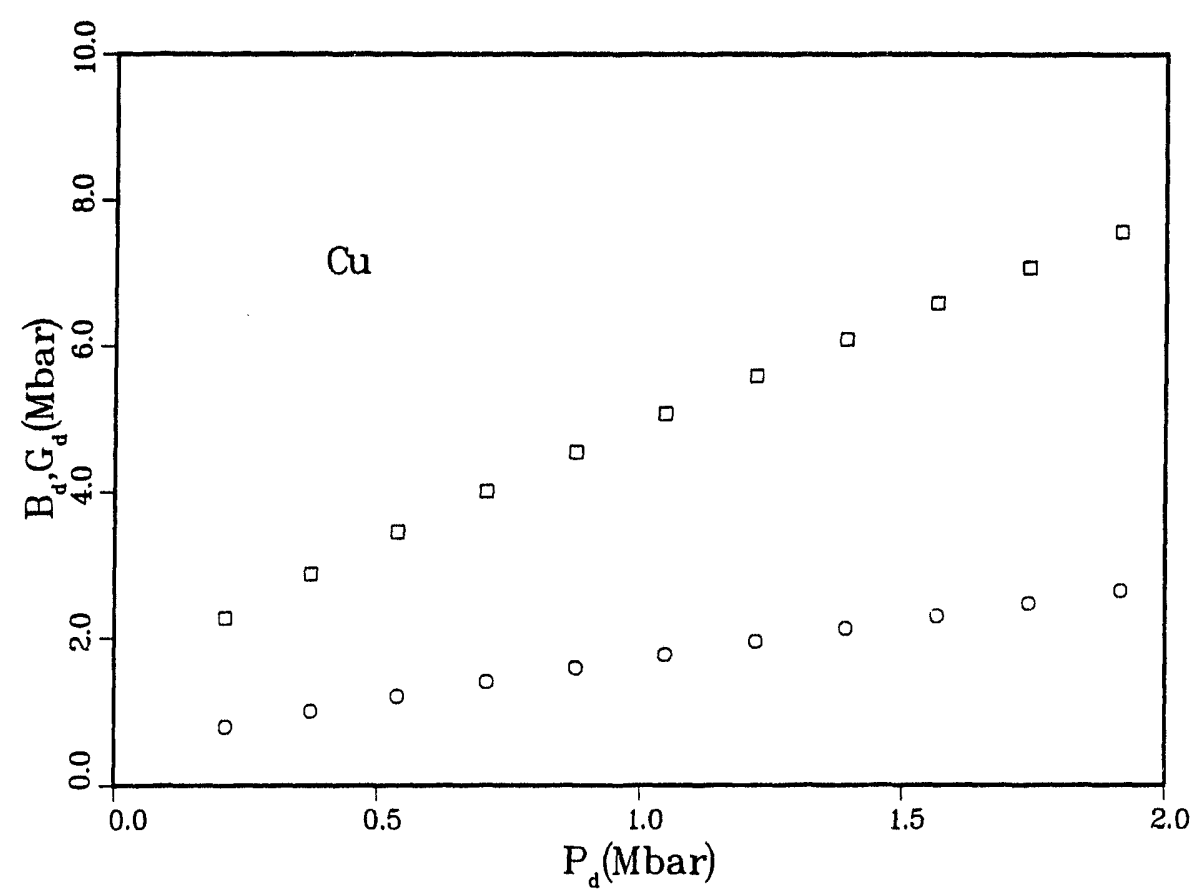

Fig. 15. The shear modulus (circles) and the bulk modulus (squares) at the point of half-final plastic strain plotted versus the pressure at this point for $\mathrm{Cu}$. These quantities are very good approximations to averages through the shock path with respect to plastic or volumetric strain.

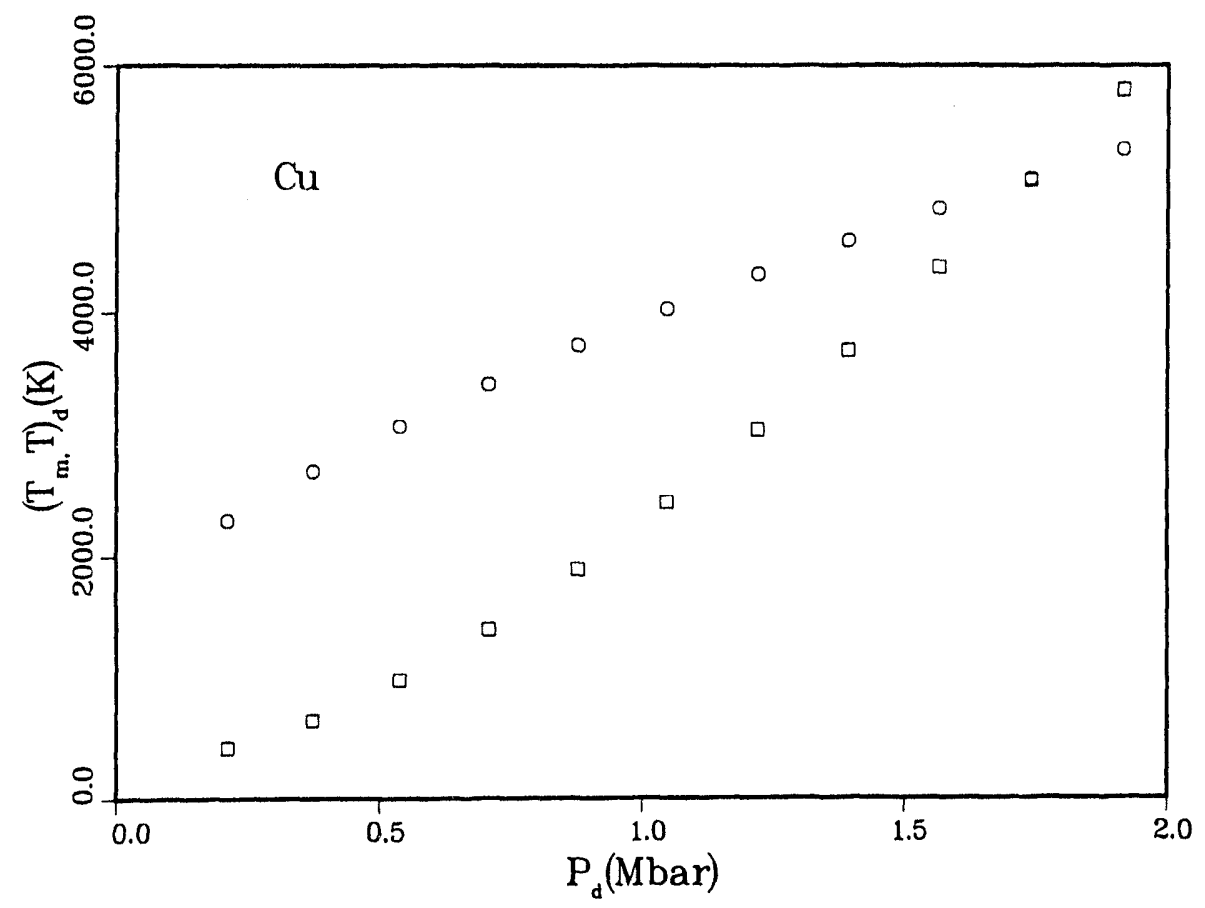

Fig. 16. The calculated melt temperature (circles) and temperature (squares) at the point of halffinal plastic strain plotted versus the pressure at this point for $\mathrm{Cu}$. 


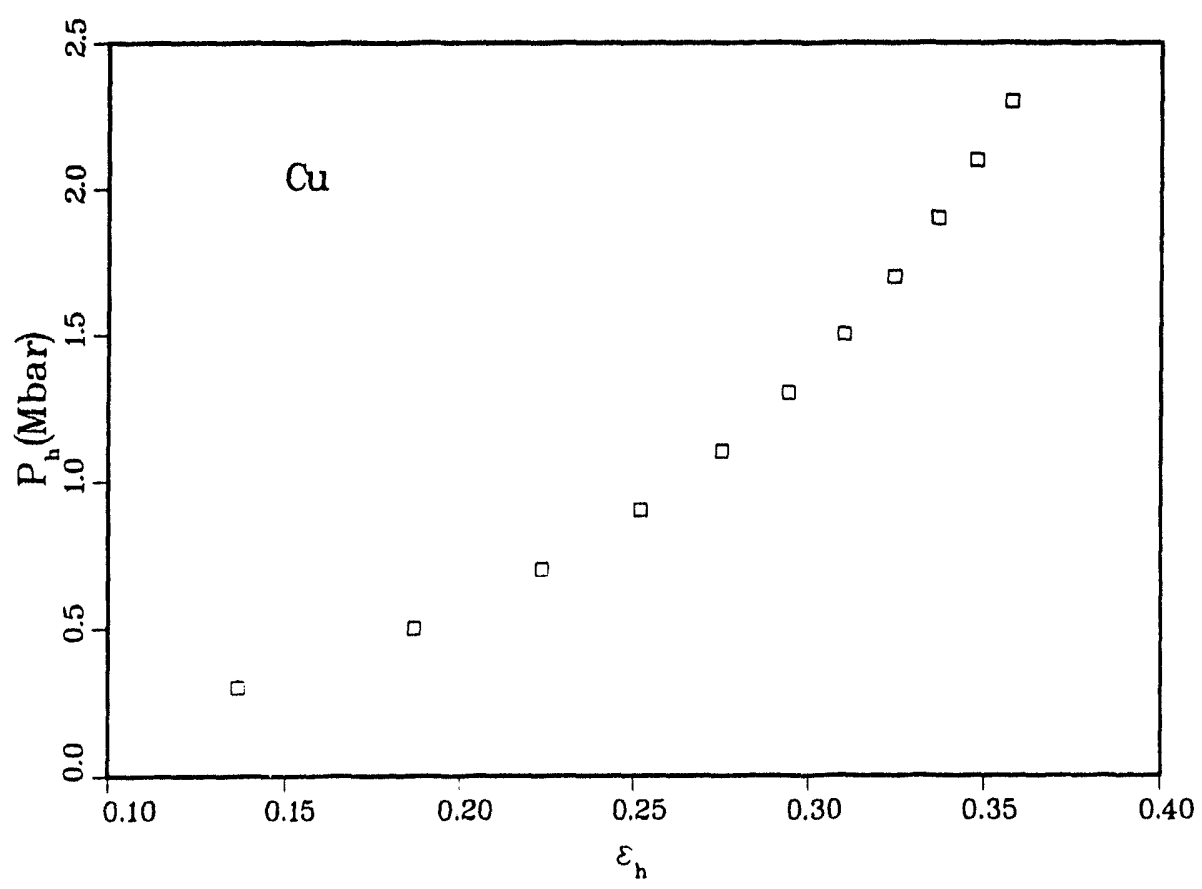

Fig. 17. Hugoniot pressure plotted versus volumetric strain for $\mathrm{Cu}$ for the same shock waves used to generate the earlier figures for $\mathrm{Cu}$.

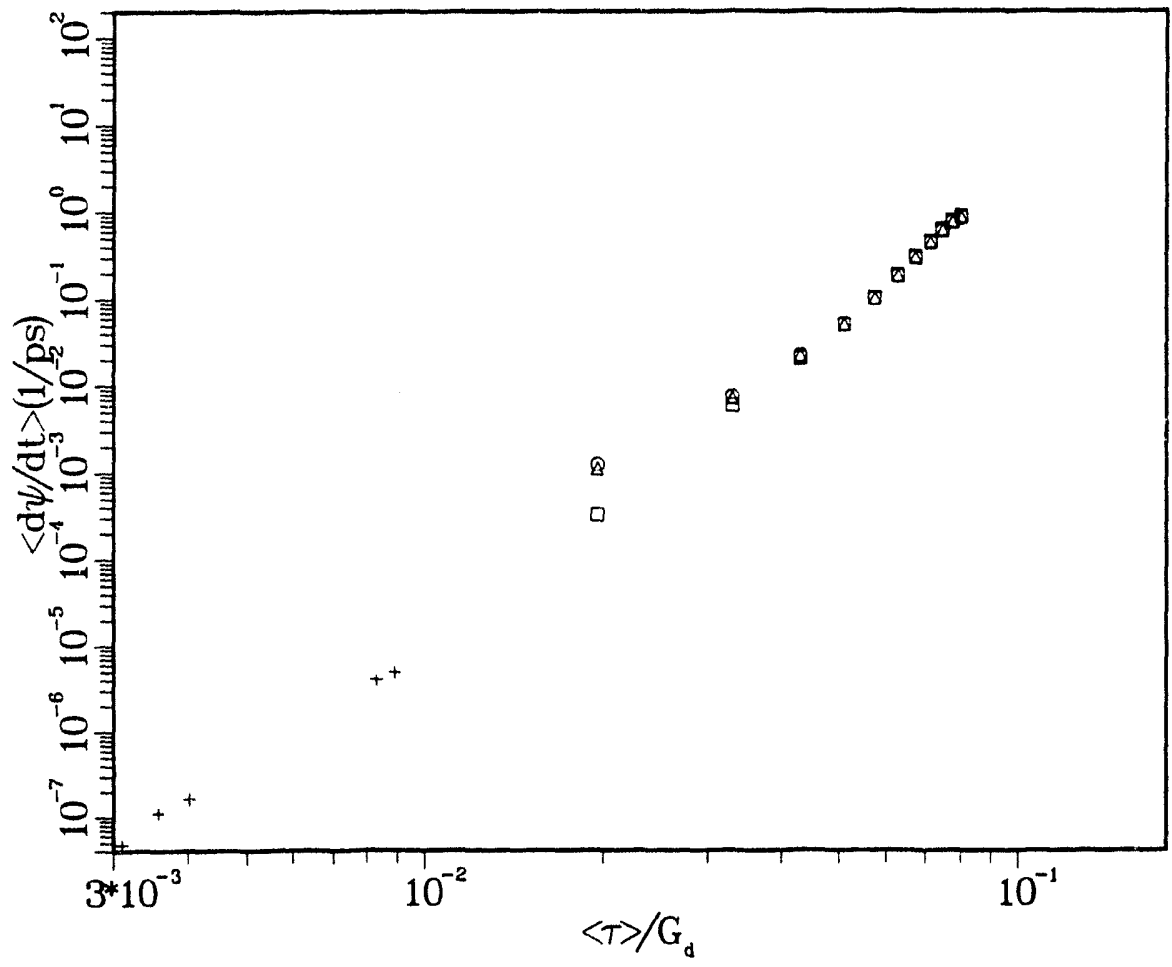

Fig. 18. Average plastic strain rates plotted versus average deviatoric stresses divided by $G_{d}$ for $\mathrm{Cu}$. Averages are through the shock path. The five pluses are pressure-shear data. The other points are the same as those of Fig. 13. 


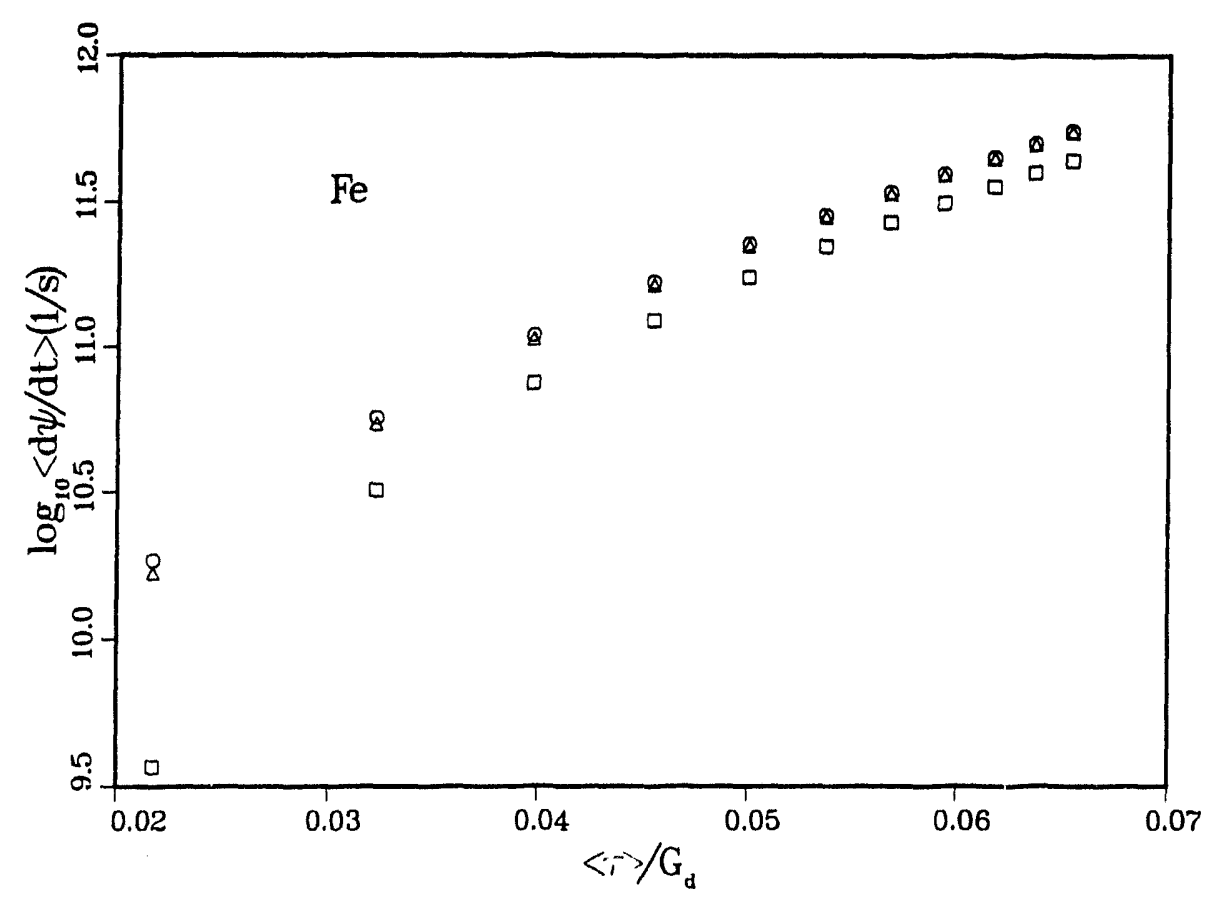

Fig. 19. Average plastic strain rate versus average deviatoric stress divided by $G_{d}$ for various shock strengths for Fe. Averages are through the shock path. The squares, triangles, and circles correspond to strain rates averaged using Eq. (21), using the volumetric strain, and using the plastic strain, respectively.

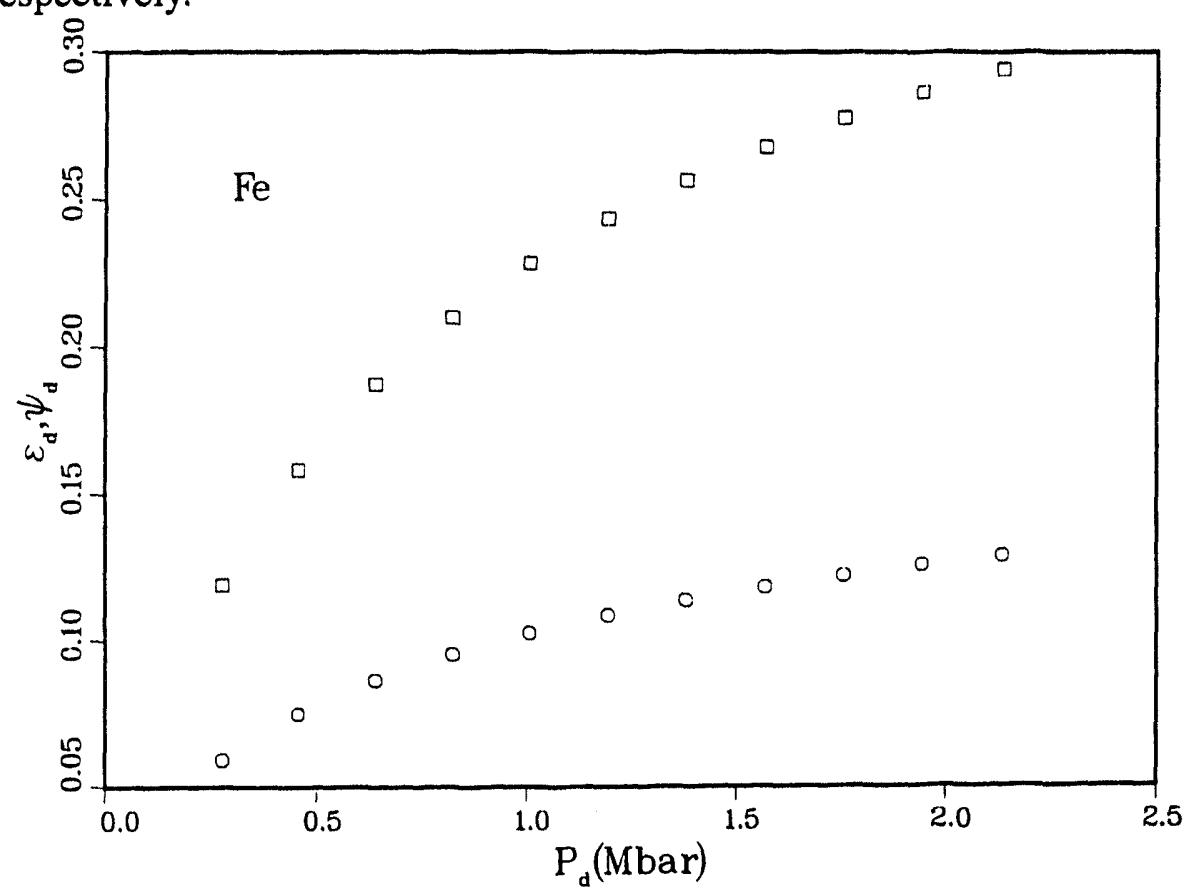

Fig. 20. Plastic strains (circles) and volumetric strains (squares) at the point of half-total plastic strain along the shock path plotted versus the pressure at this point for $\mathrm{Fe}$. 


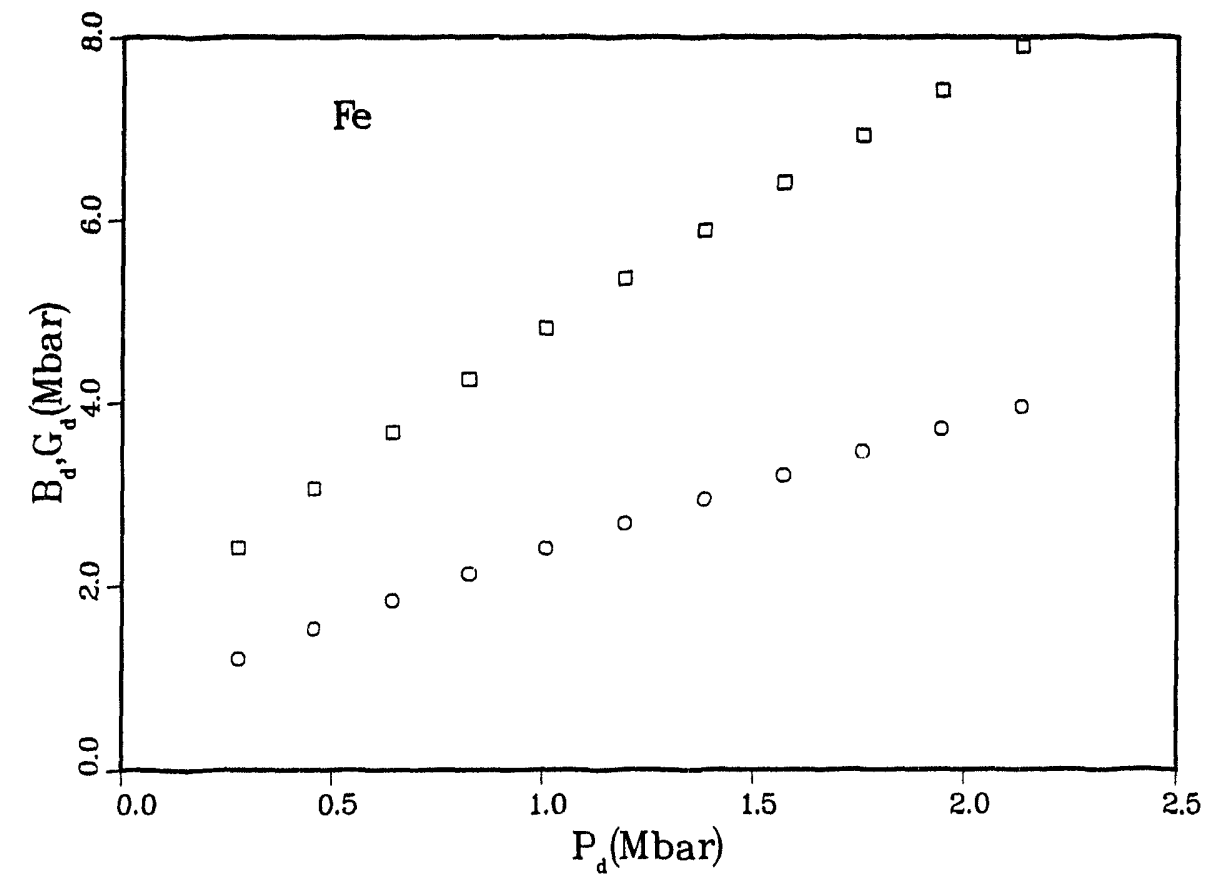

Fig. 21. The shear modulus (circles) and the bulk modulus (squares) at the point of half-final plastic strain plotted versus the pressure at this point for Fe.

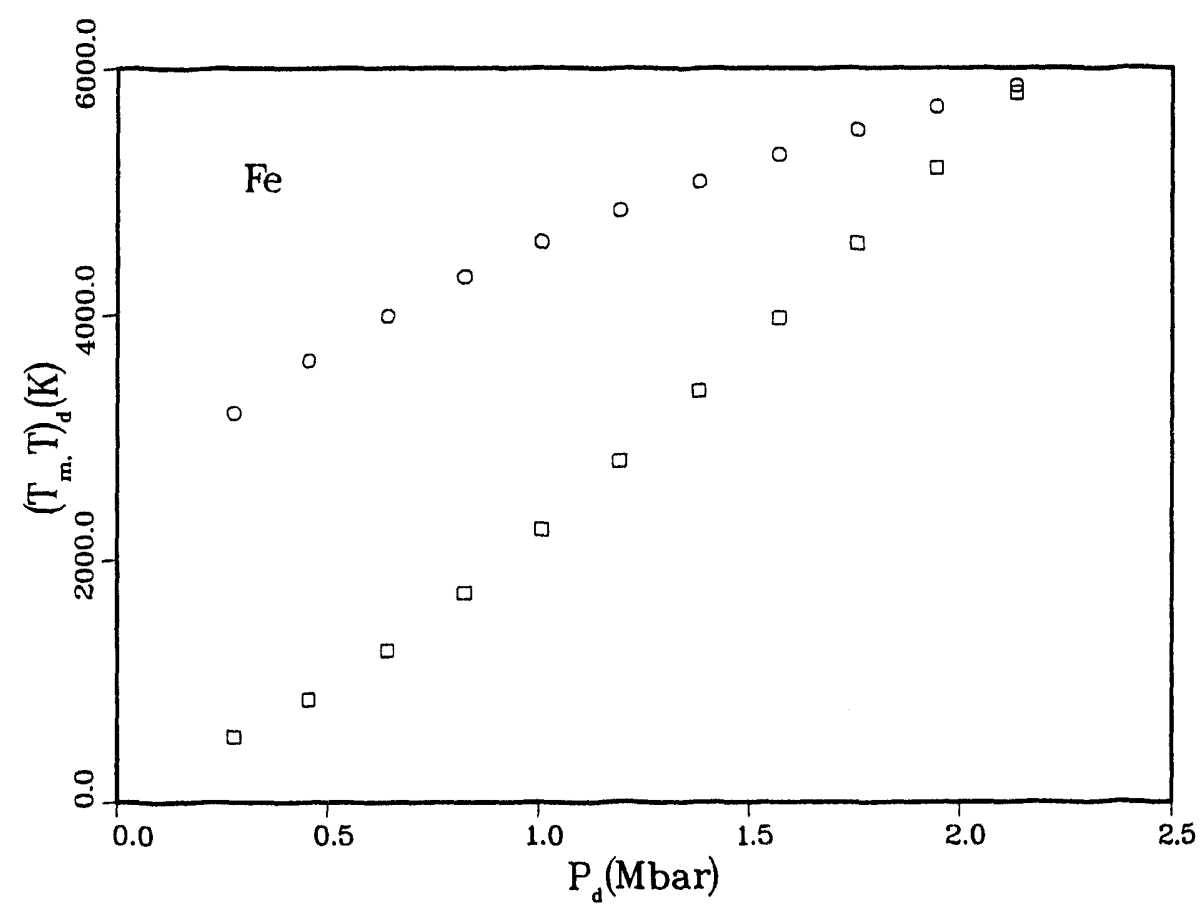

Fig. 22. The calculated melt temperature (circles) and temperature (squares) at the point of half-final plastic strain plotted versus the pressure at this point for $\mathrm{Fe}$. 


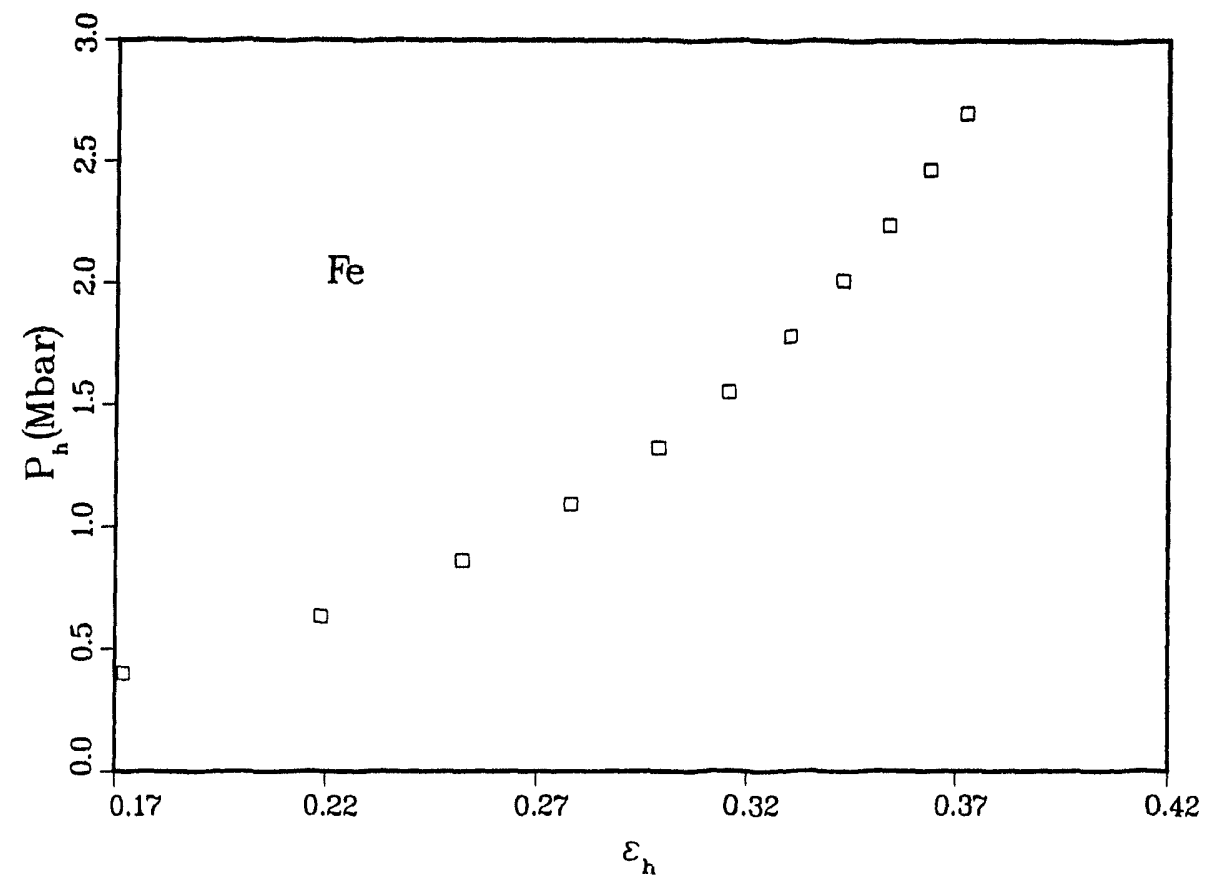

Fig. 23. Hugoniot pressure plotted versus volumetric strain for Fe for the same shock waves used to generate the earlier figures for $\mathrm{Fe}$. 
Shock Results for Tantalum

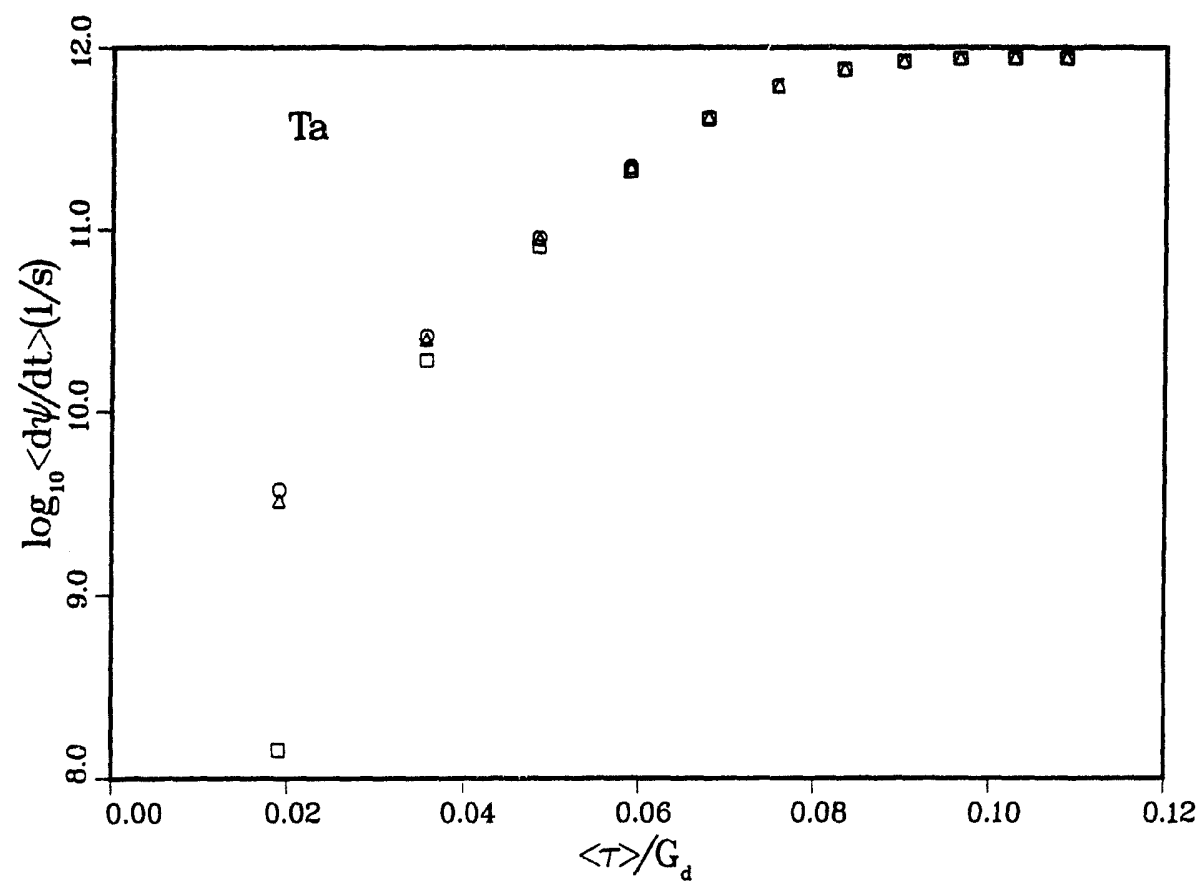

Fig. 24. Average plastic strain rate versus average deviatoric stress divided by $G_{d}$ for varicus shock strengths for Ta. Averages are through the shock path. The squares, triangles, and circles correspond to strain rates averaged using Eq. (21), using the volumetric strain, and using the plastic strain, respectively.

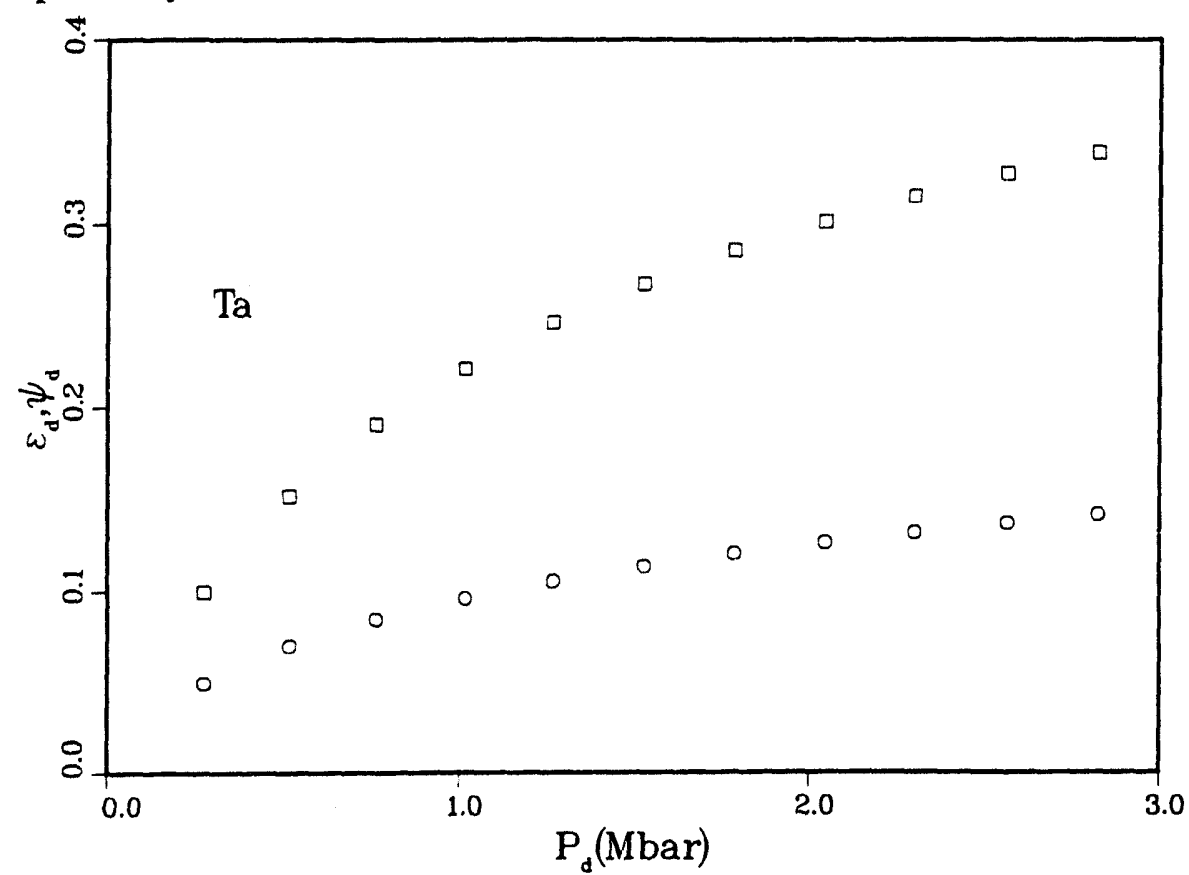

Fig. 25. Plastic strains (circles) and volumetric strains (squares) at the point of half-total plastic strain along the shock path plotted versus the pressure at this point for $\mathrm{Ta}$. 


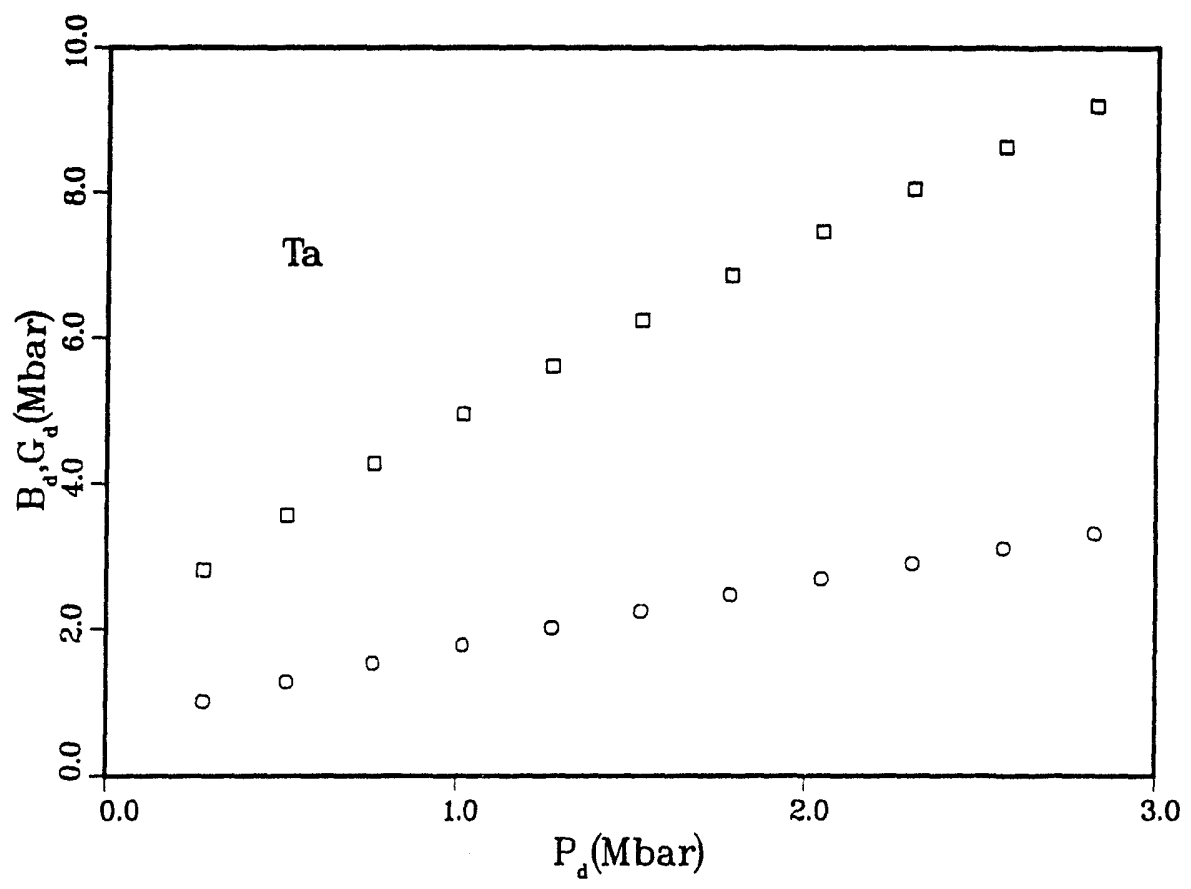

Fig. 26. The shear modulus (circles) and the bulk modulus (squares) at the point of half-final plastic strain plotted versus the pressure at this point for Ta.

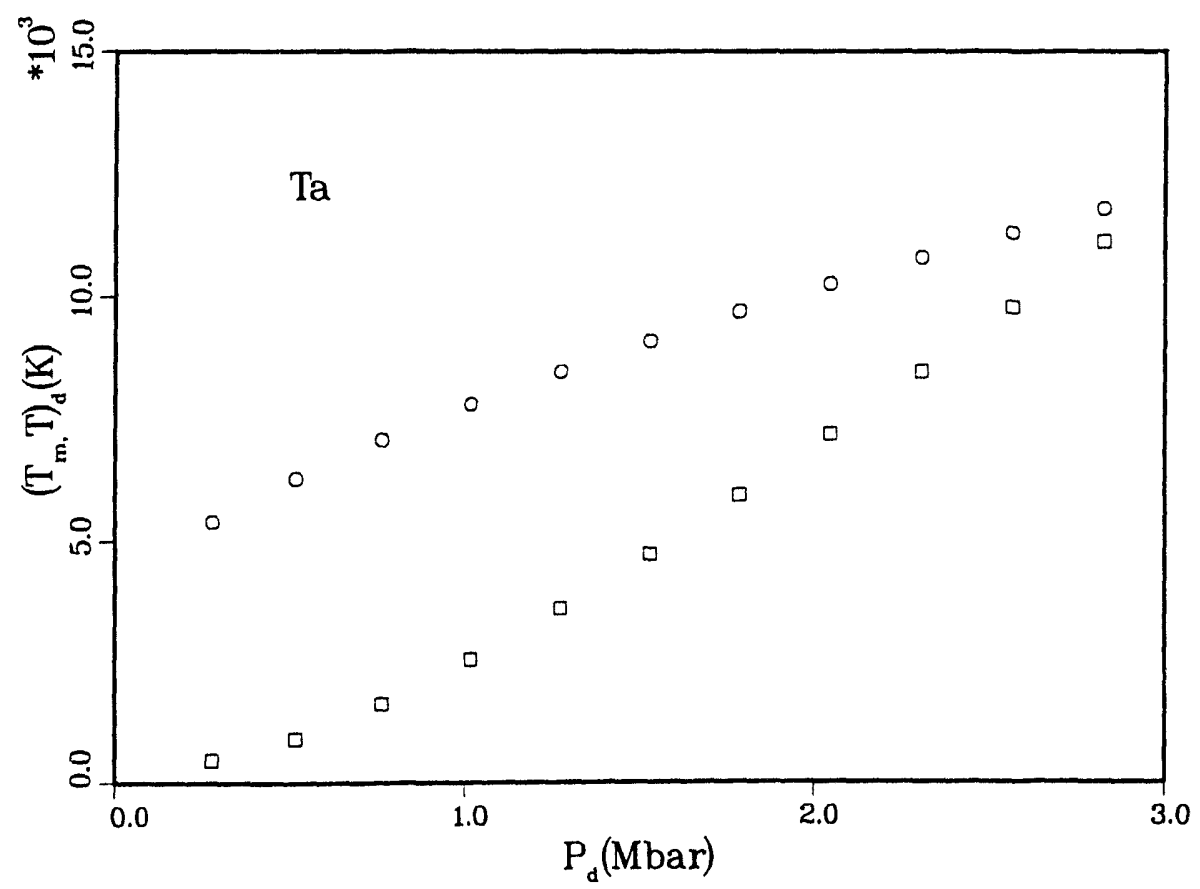

Fig. 27. The calculated melt temperature (circles) and temperature (squares) at the point of half-final plastic strain plotted versus the pressure at this point for Ta. 


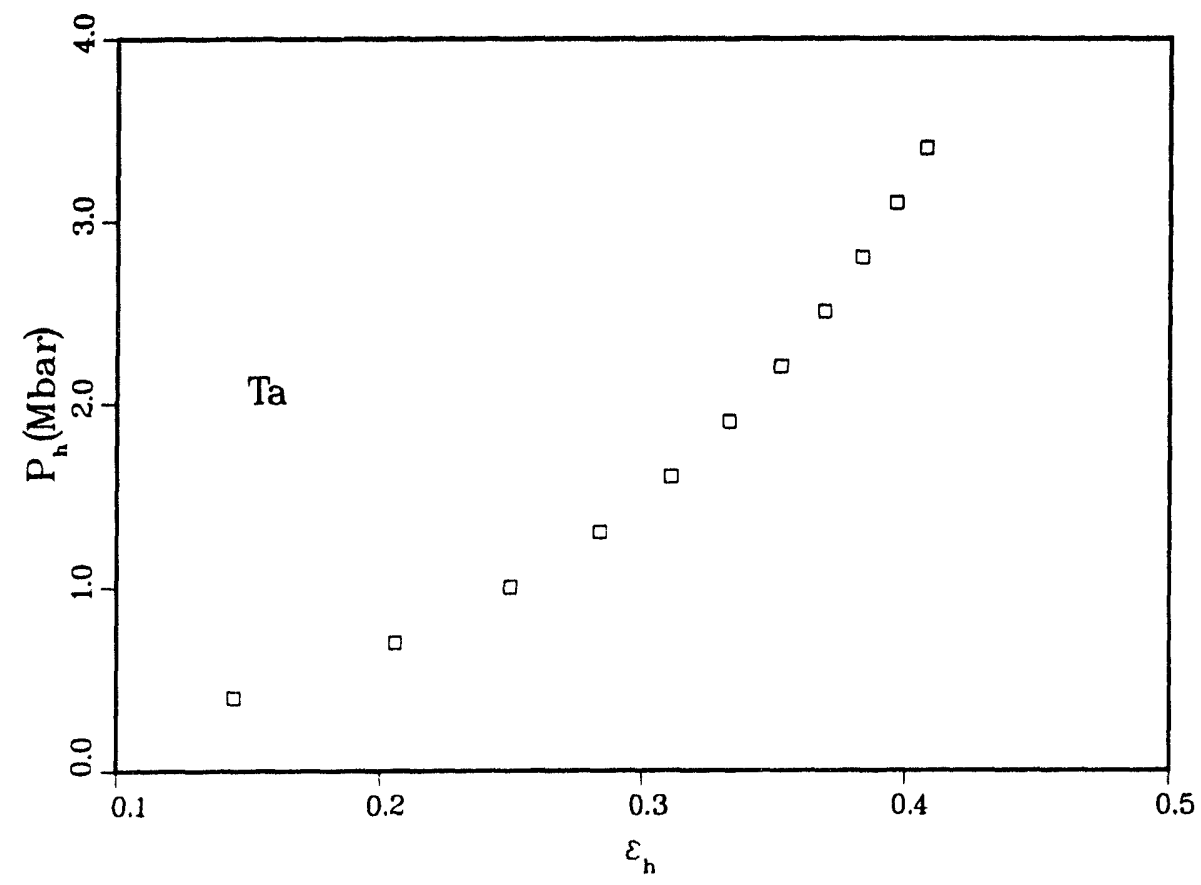

Fig. 28. Hugoniot pressure plotted versus volumetric strain for the same shock waves used to generate the earlier figures for Ta. 


\section{Shock Results for Uranium}

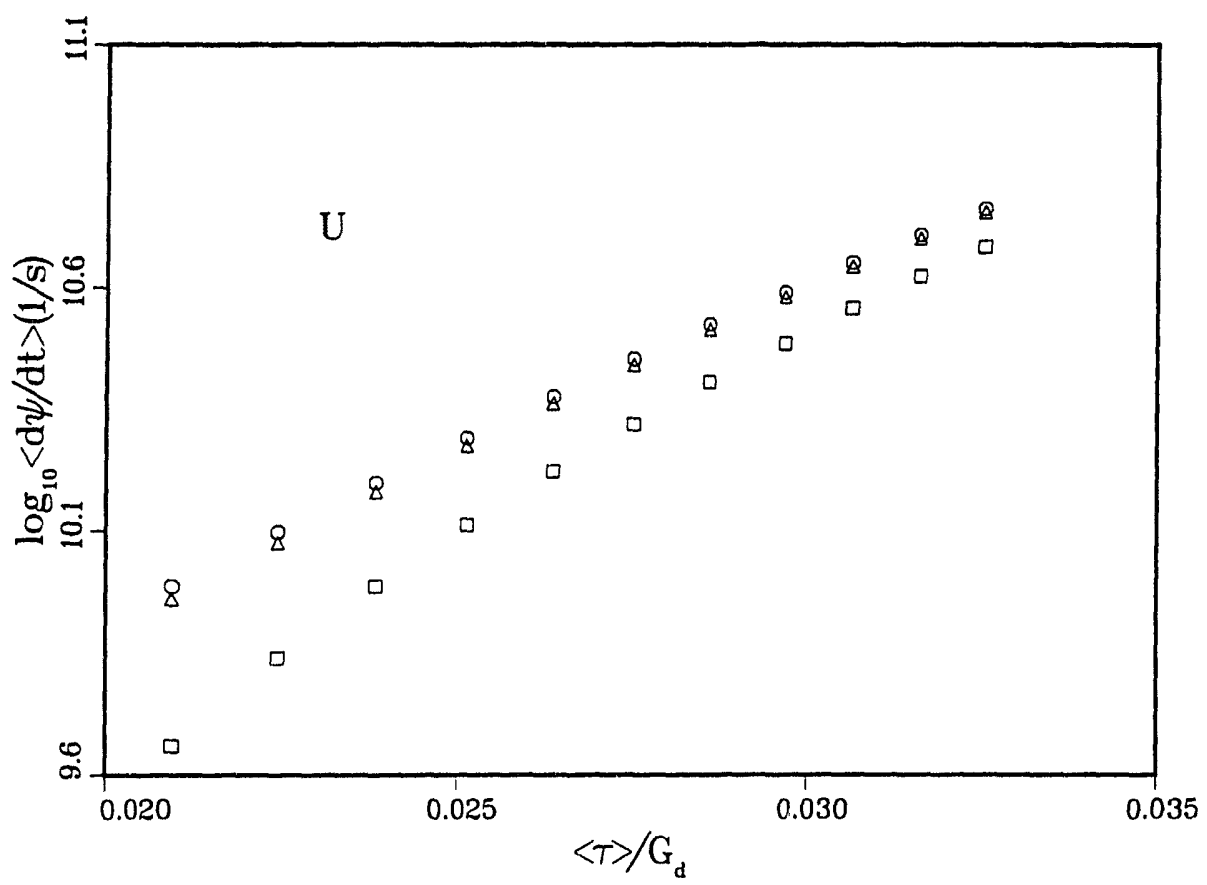

Fig. 29. Average plastic strain rate versus average deviatoric stress divided by $\mathrm{G}_{\mathrm{d}}$ for various shock strengths for U. Averages are through the shock path. The squares, triangles, and circles correspond to strain rates averaged using Eq. (21), using the volumetric strain, and using the plastic strain, respectively.

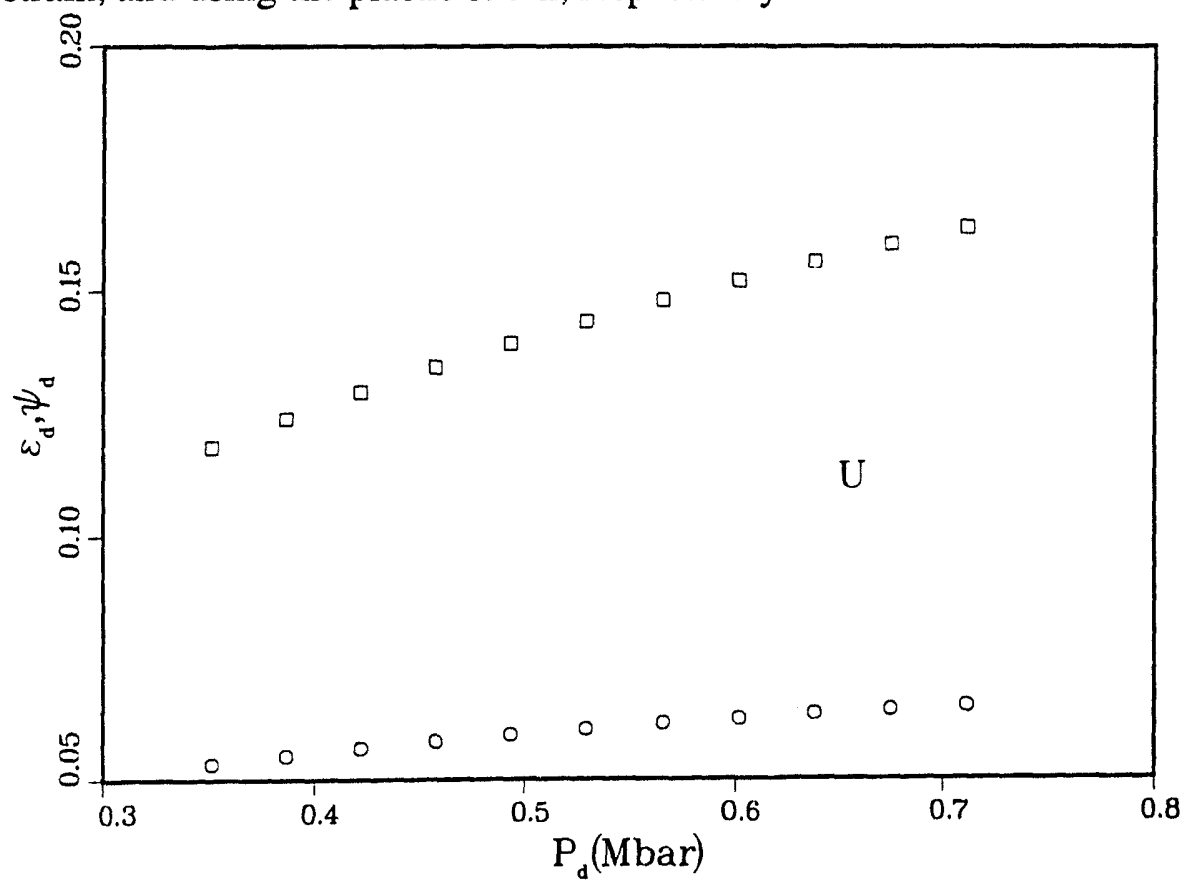

Fig. 30. Plastic strains (circles) and volumetric strains (squares) at the point of half-total plastic strain along the shock path plotted versus the pressure at this point for $U$. 


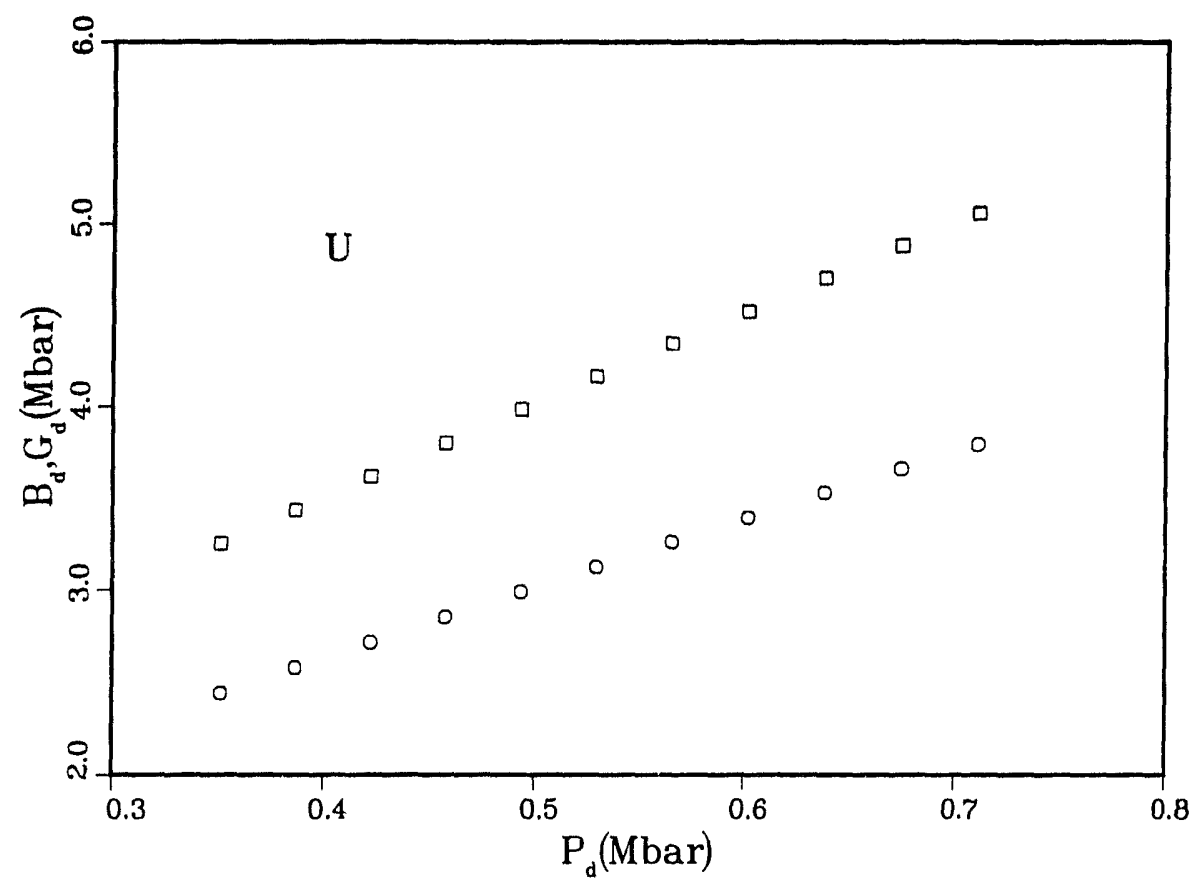

Fig. 31. The shear modulus (circles) and the bulk modulus (squares) at the point of half-final plastic strain plotted versus the pressure at this point for $U$.

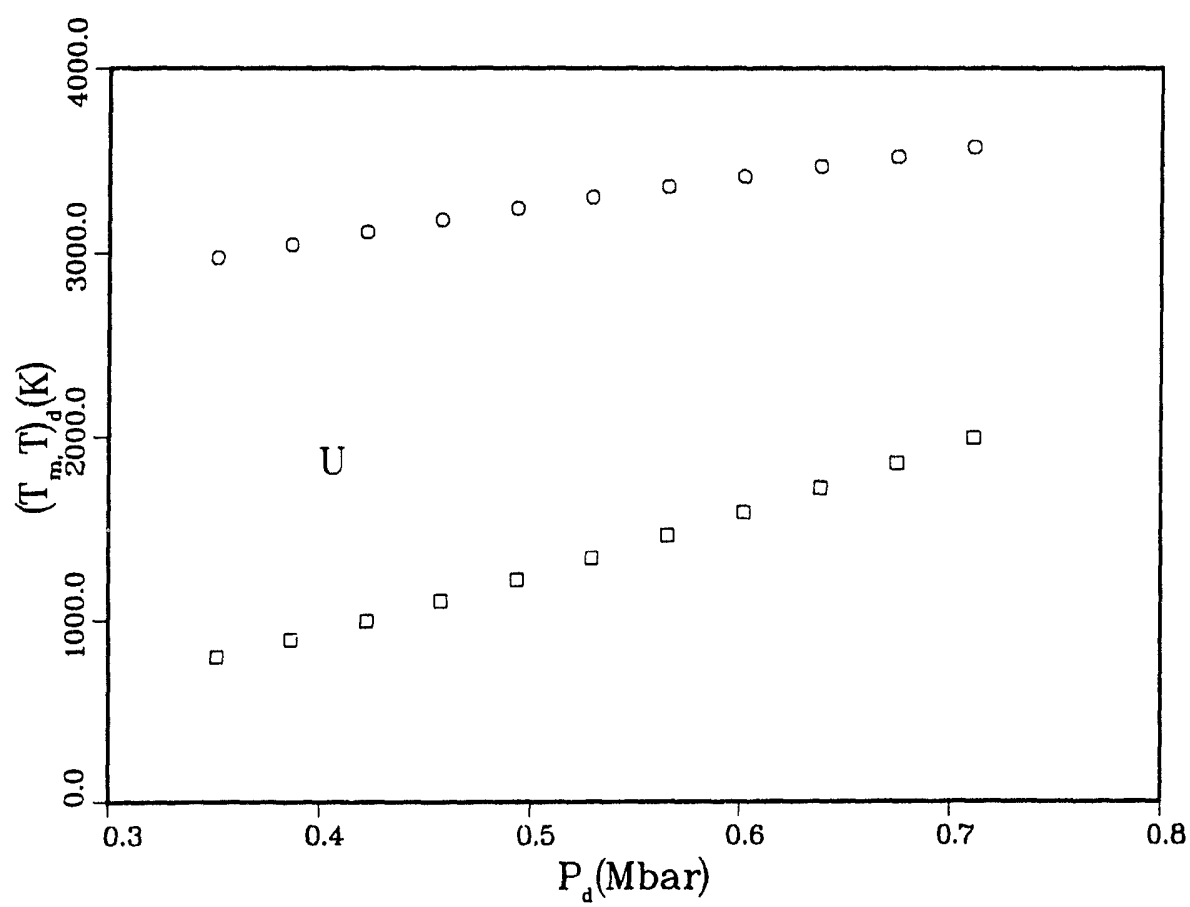

Fig. 32. The calculated melt temperature (circles) and temperature (squares) at the point of halffinal plastic strain plotted versus the pressure at this point for $U$. 


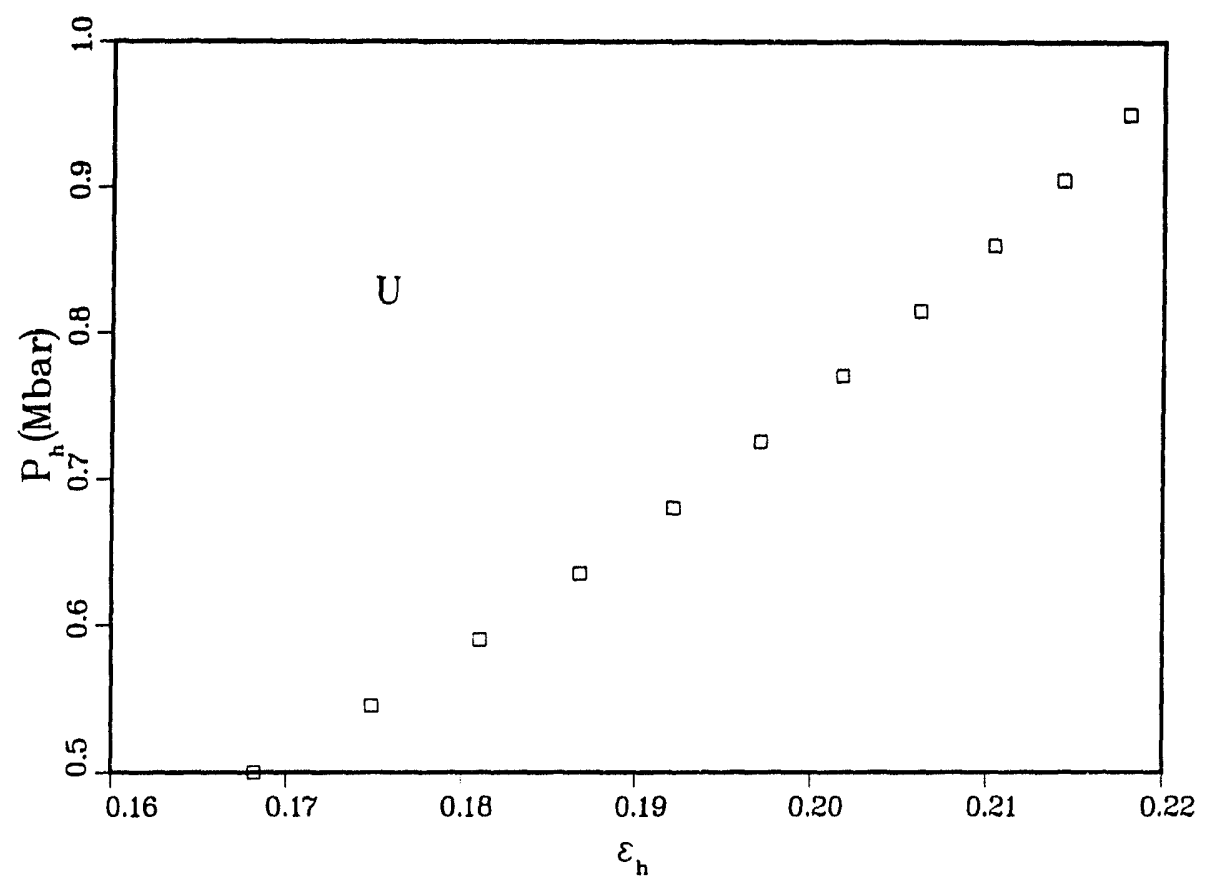

Fig. 33. Hugoniot pressure plotted versus volumetric strain for the same shock waves used to generate the earlier figures for $U$.

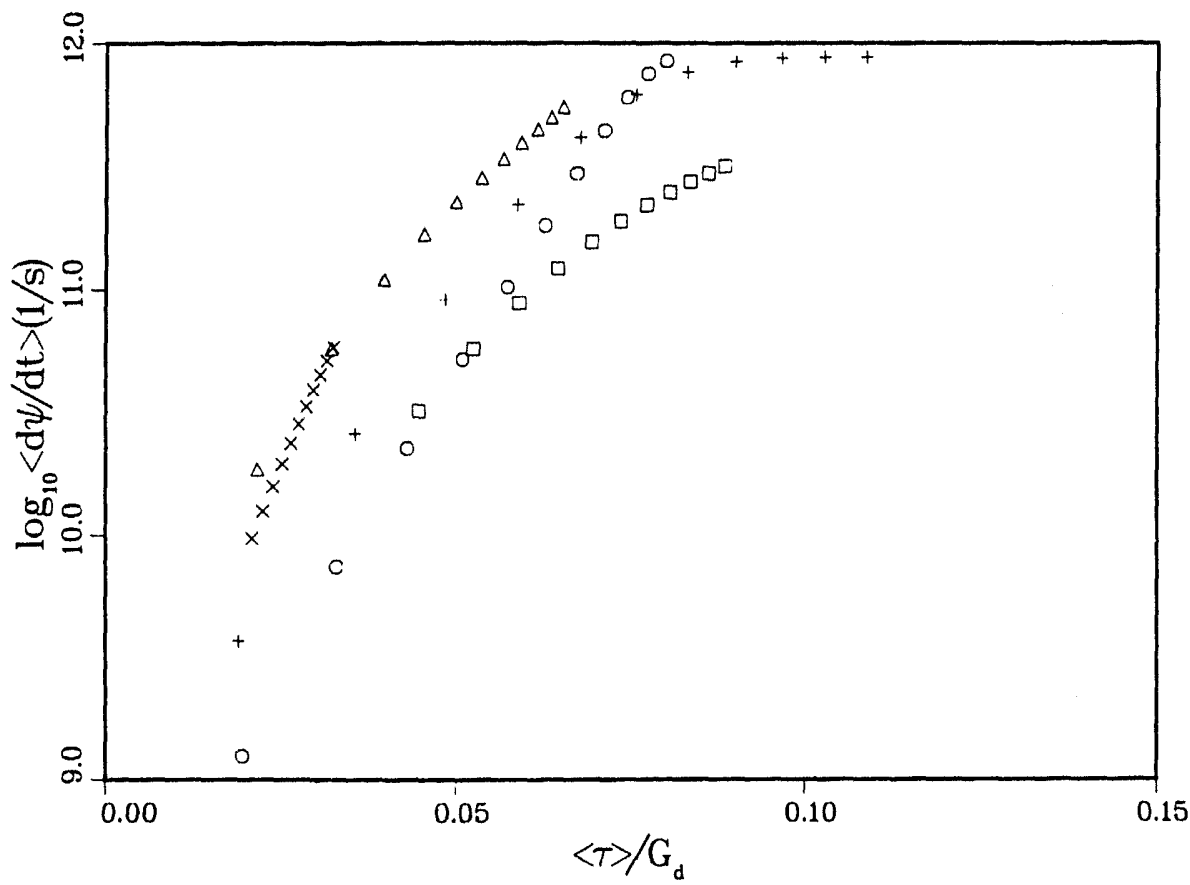

Fig. 34. Average plastic strain rate $\left(\langle\dot{\psi}\rangle_{\psi}\right)$ versus average deviatoric stress divided by $\mathrm{G}_{\mathrm{d}}$ for various shock strengths for the six metals. Averages are through the shock path using the plastic strain. The squares, circles, triangles, pluses, and X's correspond to $2024 \mathrm{Al}, \mathrm{Cu}, \mathrm{Fe}, \mathrm{Ta}$, and U, respectively. 
Figures 24 through 28 show results for tantalum. These were somewhat sensitive to variations in $\gamma$ but not enough to warrant concern.

Figures 29 through 33 show results for uranium. These results were fairly robust against variations in physical parameters in the calculation.

Figure 34 shows $\langle\dot{\psi}\rangle_{\psi}$ plotted versus $\langle\tau\rangle / G_{d}$ for all the metals together. This plot shows the degree of universality exhibited by these quantites.

\section{Conclusions}

Calculated results using Wallace's strong shock theory are given in this document for a variety of metals. The results are not as firm as one would wish due to lack of complete knowledge of thermoelasticity at megabar pressures. However, the underlying theory is sound and the error analysis shows acceptable sensitivity to the thermoelastic modeling. These two features, coupled with the opportunity of obtaining for the first time a glimpse of strain-rate plasticity effects under these extreme conditions, justifies this effort to make these results more widely available.

\section{Acknowledgment}

I would like to thank D. C. Wallace for providing guidance and access to research notebooks. This work is very much indebted to his earlier effort. 


\section{References}

${ }^{1}$ D. C. Wallace, Phys. Rev. B24, 5597 (1981).

${ }^{2}$ D. C. Wallace, Phys. Rev. B24, 5607 (1981).

${ }^{3}$ D. C. Wallace, Phys. Rev. B22, 1477 (1980).

${ }^{4}$ D. C. Wallace, Phys. Rev. B22, 1487 (1980).

${ }^{5}$ D. C. Wallace, private communication.

${ }^{6}$ G. K. Straub and W. Harrison, Phys. Rev. B31, 7668 (1985).

${ }^{7}$ V. L. Moruzzi, J. F. Janak, and A. R. Williams, Calculated Electronic Properties of Metals, (Pergamon, N. Y., 1978).

${ }^{8}$ D. A. Boness, J. M. Brown, and A. K. McMahan, Phys. Earth and Planetary Interiors 42, 227 (1986).

${ }^{9} \mathrm{O}$. Eriksson, private communication.

${ }^{10}$ D. C. Wallace, Entropy of Liquid Metals and Melting of Elements, Proc. R. Soc. Lond. A 433, 615 (1991) and Proc. R. Soc. Lond. A433, 631 (1991).

${ }^{11}$ A. C. Mitchell and W. J. Nellis, J. Appl. Phys. 52, 3363 (1981).

${ }^{12}$ R. G. McQueen, S. P. Marsh, J. W. Taylor, J. N. Fritz, and W. J. Carter, in High - Velocity Impact Phenomena, edited by R. Kinslow (Academic, N. Y., 1970), p. 293.

13 J. M. Brown and R. G. McQueen, J. of Geophys. Research 91, 7485 (1986).

${ }^{14}$ Group GMX-6, Los Alamos Report LA-4167-MS, May, 1969.

${ }^{15} \mathrm{~S}$. Huang and R. J. Clifton, in Proceedings of the IUTAM Symposium on Macro - and MicroMechanics of High Velocity Deformation and Fracture, Tokyo, Japan, 1985. 

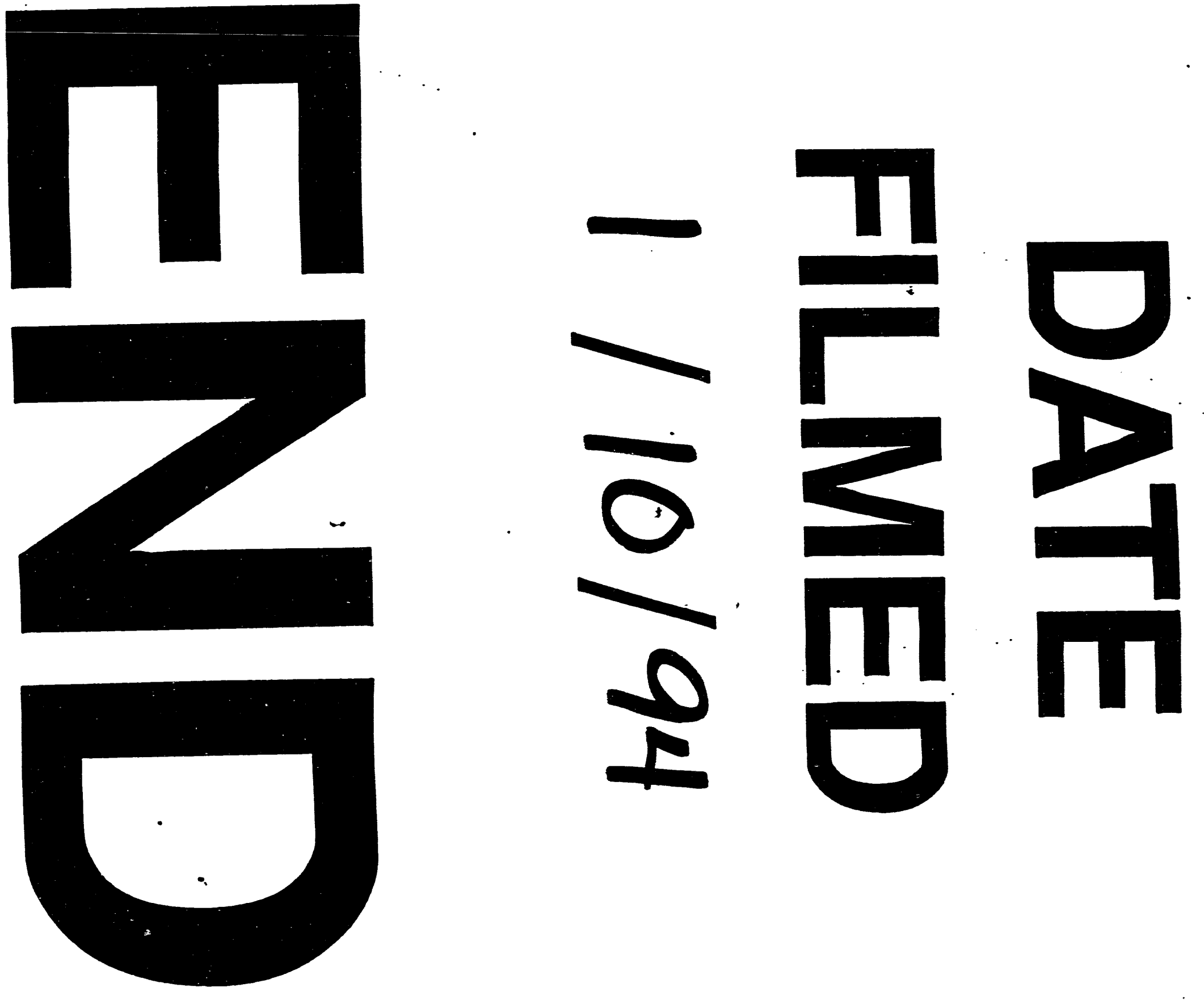


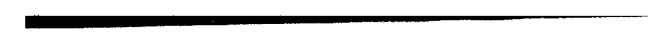

